City University of New York (CUNY) CUNY Academic Works

2-2016

\title{
The Effect of the Long Anchor Duration on Performance in the Temporal Bisection Procedure
}

Sho Araiba

Graduate Center, City University of New York

\section{How does access to this work benefit you? Let us know!}

More information about this work at: https://academicworks.cuny.edu/gc_etds/681

Discover additional works at: https://academicworks.cuny.edu

This work is made publicly available by the City University of New York (CUNY).

Contact: AcademicWorks@cuny.edu 


\title{
THE EFFECT OF THE LONG ANCHOR DURATION ON PERFORMANCE IN THE TEMPORAL BISECTION PROCEDURE
}

\author{
by
}

\section{SHO ARAIBA}

A dissertation submitted to the Graduate Faculty in Psychology in partial fulfillment of the requirements for the degree of Doctor of Philosophy, The City University of New York. 
(C) 2016

Sho Araiba

All Rights Reserved

ii 
This manuscript has been read and accepted for the Graduate Faculty in Psychology to satisfy the dissertation requirement for the degree of Doctor of Philosophy

Dr. Bruce L. Brown

Date

Chair of Examining Committee

Dr. Maureen O'Connor

Date

Executive Officer

Dr. Nancy S. Hemmes

Dr. Robert N. Lanson

Dr. Valérie Doyère

Dr. Robert W. Allan

Supervisory Committee

THE CITY UNIVERSITY OF NEW YORK

iii 
Abstract

THE EFFECT OF THE LONG ANCHOR DURATION ON PERFORMANCE IN THE

TEMPORAL BISECTION PROCEDURE

by

SHO ARAIBA

\section{Adviser: Professor Bruce L. Brown}

The present study investigated the determinants of the location of the bisection point (BP) in the temporal bisection procedure. Interest in the study of temporal perception emerged from the finding of the scalar property such as Weber's law for time and the ratio rule in behavior under Pavlovian and instrumental conditioning. One method to investigate temporal perception in both non-human animals and humans is the temporal bisection procedure. The primary dependent variable of this procedure is the BP, which has been taken as the subjective midpoint between two durations. The ratio similarity rule (Gibbon, 1981) hypothesizes that the location of the BP is determined by a ratio comparison between the short anchor duration and the long anchor duration, and the midpoint is at the geometric mean (GM) of the two anchors. The Short/no short hypothesis, on the other hand, indicates that the location of the BP is only influenced by the short duration (Machado \& Keen, 2003). The present study investigated how the value of the long anchor duration influenced the BP in the temporal bisection procedure. In Exp. 1, pigeons were exposed to different values of the long anchor duration across four experimental phases. The four phases were a 3- vs. 9-s condition, 3- vs. 27-s condition, 3-vs. 13.5-s condition, and 3- vs. 27-s condition. In the bisection test, durations that were intermediate between the anchor durations were also presented. The stimulus duration that occasioned a 'long' choice response on 50\% of 
the trials, $\mathrm{p}($ long $)=0.5$, defined the BP. The results showed that the BP fell at the GM of two anchor durations in each phase as predicted by the ratio similarity rule. This outcome fails to support the Short/no short hypothesis even though the anchor pairs of the first two phases of the experiment were the same values as in Machado and Keen (2003; Exp. 3). Exp. 2 was conducted to further assess the results of Exp. 1 by counterbalancing the sequence of conditions. Two groups of pigeons were exposed to two discrimination conditions, 3- vs. 9-s and 3-vs. 27-s, in opposite orders. The results showed that within groups the location of the BP differed between the 3- vs. 9-s condition and the 3- vs. 27-s condition as in Exp. 1. However, the degree of the change in the BP differed between groups. The difference was larger when the 3- vs. 9-s condition was followed by the 3- vs. 27-s condition (Group 3-9 to 3-27) than when the 3- vs. 27s condition was the initial phase (Group 3-27 to 3-9). In addition, for Group 3-9 to 3-27, the BP was at the GM of the two anchor durations in the 3-vs. 27-s condition when the initial training condition was 3-vs. 9-s as predicted by the ratio similarity rule and suggesting a relatively strong influence of the long anchor duration. On the other hand, for Group 3-27 to 3-9, the BP in the 3vs. 27-s condition was less than the GM when it was the initial training condition, which indicated that the effect of the long duration was relatively small, conforming more closely to the Short/no short hypothesis. Thus, the location of the BP in the 3- vs. 27-s condition depended on the order of conditions. Keypecking to the sample key during a trial, sample-cue responding, was also examined. The functions relating sample-cue responding to elapsed trial time were similar across phases for each group. That is, even though there was a difference in the shape of the functions between the groups, the pattern of sample-cue responding in the initial phase did not change in the second phase within each group. The degree of superposition of the functions across two phase was better with a relative-time scale than with an absolute-time scale for Group 
3-9 to 3-27, showing the scalar property of sample-cue responding. For Group 3-27 to 3-9, the degree of superposition was better with the absolute-time scale than with the relative-time scale. The results of the present study suggested that the initial discrimination condition established different degrees of stimulus control of behavior by the long duration, and that the initiallyacquired stimulus control transferred to the following discrimination condition. The implication of this interpretation to other research findings was discussed. 


\section{Acknowledgements}

I would like to express my deepest gratitude to my advisor, Dr. Bruce L. Brown, for mentorship he provided throughout my time in the Learning Processes and Behavior Analysis program / Behavior Analysis training area. Especially the time he offered me to shape my analytic, scientific and verbal behavior one-on-one every Friday for the first three years in this program became both the foundation of my professional activities as well as good memory.

I would also like to thank my committee members, Drs. Nancy S. Hemmes, Robert N. Lanson, Valérie Doyère, Robert W. Allan and Lanny Fields for their valuable advice, insights and additional research opportunities at various points in time throughout this program. I really appreciate their time and support to serve on my doctoral committee.

I am grateful to the faculty, students, administrative staff and vivarium staff affiliated with the Queens College Psychology Department and the Graduate Center Psychology Department. I also want to thank my colleagues in this program, especially those in the BrownHemmes laboratory: Drs. Erich Grommet, Catherine Tsiris, Joseph Jacobs, Roxana Nedelcu, and Kathleen Mangiapanello, and Daniel Garces, Allison Hirsch, Jennifer Morrison, Parvene Farhoody and my pigeons. You have always stimulated me intellectually and motivated me throughout the time in this program.

I would like to thank all the support that I have received from my father Kiyotaka, my mother Toyomi, and my sister Moe, not only during this program but also throughout my entire college education in New York. Finally, I would like to express my love to my wife, Mariko, for kicking my butt to finish up this dissertation! 


\section{Table of Contents}

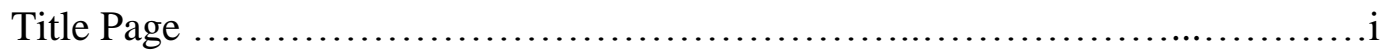

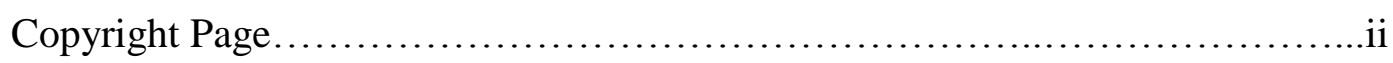

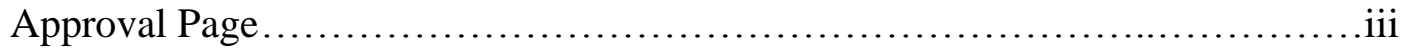

Abstract ............................................................

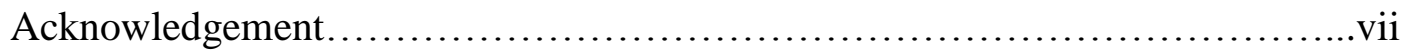

Table of Contents........................................................ vii

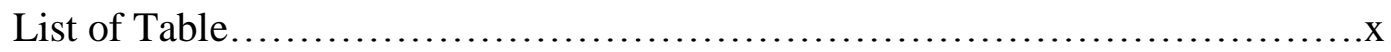

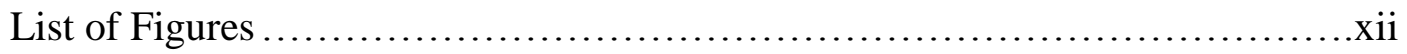

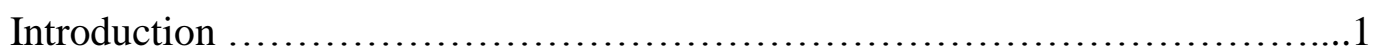

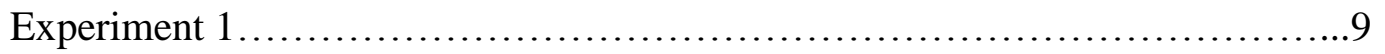

Method............................................................... 10

Subjects.................................................. 10

Apparatus...................................................... 10

Experimental Design..........................................11

Procedure..........................................................11

Data Analysis............................................... 15

Results............................................................. 16

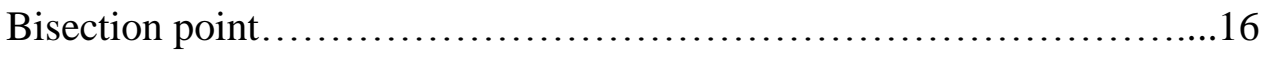

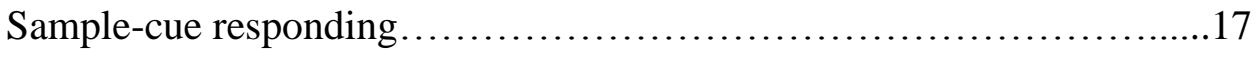

Discussion......................................................... 19

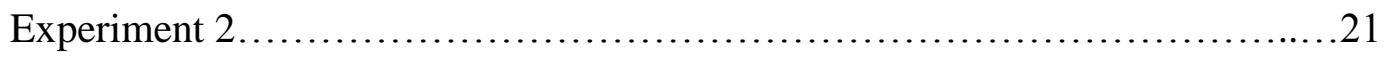

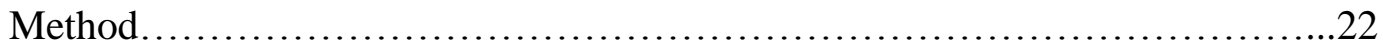


Subjects 22

Apparatus.....................................................22

Experimental Design.......................................22

Procedure...........................................................

Data Analysis.................................................24

Results................................................................

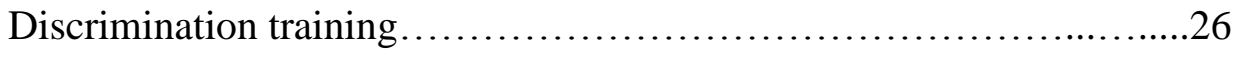

Psychometric function..........................................27

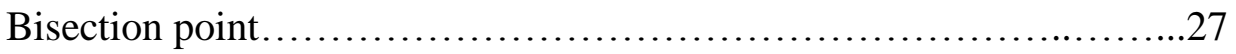

Gamma.................................................28

PLM prediction...............................................29

Sample-cue responding.....................................29

Psychometric functions in relative time........................... 34

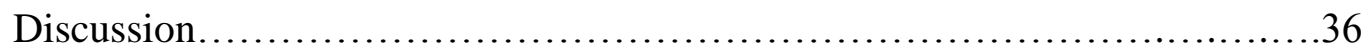

General Discussion................................................. 38

Sessions to Acquisition.........................................39

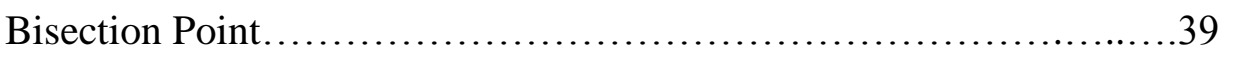

Sample-cue responding.....................................42

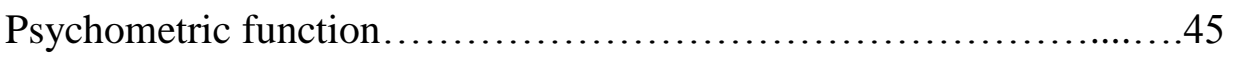

Implications..............................................45

Conclusion...................................................49

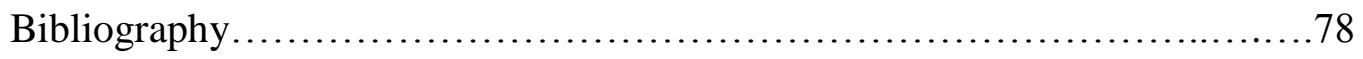




\section{Lists of Tables}

Table 1. The scheme of Experiment 1 for two groups (rows) and four phases......52

Table 2. Group x Phase mixed analysis of variance of BP in the four phases of Exp. 1

.53

Table 3. The scheme of Experiment 2 for two groups (rows) and two phases ......54

Table 4. The bisection point (BP), the geometric mean (GM), and the harmonic mean (HM) in each phase for each group of Experiment 2 ..................55

Table 5. Group $x$ Phase $x$ Time analysis of variance of normalized rate for the sample-cue response for an absolute time scale in Exp. 2......................56

Table 6. $\quad$ Phase $x$ Time analysis of variance of normalized rate for the sample-cue response for an absolute time scale for Group 3-9 to 3-27 in Exp. 2................57

Table 7. Phase $x$ Time analysis of variance of normalized rate for the sample-cue response for an absolute time scale for Group 3-27 to 3-9 in Exp. 2...............58

Table 8. Group $x$ Phase $x$ Time analysis of variance of normalized rate for the sample-cue

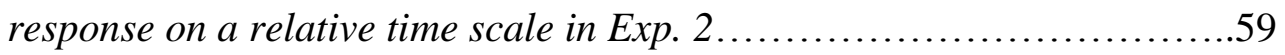

Table 9. $\quad$ Phase $x$ Time analysis of variance of normalized rate for the sample-cue response for a relative time scale for Group 3-9 to 3-27 in Exp. 2................60

Table 10. $\quad$ Phase $x$ Time analysis of variance of normalized rate for the sample-cue response for a relative time scale for Group 3-27 to 3-9 in Exp. 2................61

Table 11. Group $x$ Phase $x$ Time analysis of variance of $p$ (long) for the psychometric

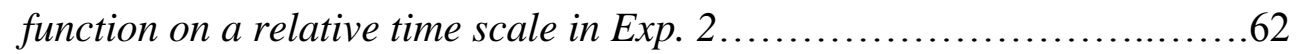

Table 12. $\quad$ Phase $x$ Time analysis of variance of $p$ (long) for the psychometric function on a relative time scale for Group 3-9 to 3-27 in Exp. 2. 
Table 13. $\quad$ Phase $x$ Time analysis of variance of $p$ (long) for the psychometric function on a relative time scale for Group 3-9 to 3-27 in Exp. 2.....................64 


\section{Lists of Figures}

Figure 1. Hypothetical performance of a participant plotted as the probability of a 'long' response as a function of duration on a log scale in the temporal bisection

procedure .65

Figure 2. The group mean bisection point $( \pm S E M)$ as a function of phases for both groups in Exp. 1 .66

Figure 3. Group mean responses per second $( \pm S E M)$ as a function of elapsed time of the long anchor stimulus for the average across three groups in Exp. 1

Figure 4. Group mean number of sessions to mastery criterion $( \pm S E M)$ as a function of phases for Group 3-9 to 3-27 and Group 3-27 to 3-9 in Exp. 2............68

Figure 5. Group mean probability of a "Long" response $( \pm S E M)$ in each phase as a function of stimulus duration in Exp. 2.

Figure 6. Group mean bisection point $( \pm S E M)$ as a function of phase for Group 3-9 to 3-27 and for Group 3-27 to 3-9 in Exp. 2. ...............................70

Figure 7. Group mean gamma $( \pm S E M)$ as a function of phase for Group 3-9 to 3-27 and Group 3-27 to 3-9 in Exp. 2. 71

Figure 8. Bisection point (obtained) plotted against bisection point (PLM) for each phase of each group in Exp. 2 72

Figure 9. Group mean responses per second $( \pm S E M)$ on the long anchor sample cue as a function of elapsed time (s) for two phases in Exp. $2 \ldots \ldots \ldots \ldots \ldots \ldots \ldots . \ldots 73$

Figure 10. Group mean normalized response rate $( \pm S E M)$ at the sample key as a function of time during the first $9 \mathrm{~s}$ of the long anchor trials for each phase in Exp. 2. 
Figure 11. Group mean normalized response rate $( \pm S E M)$ as a function of relative elapsed time of the long anchor trials for both phases in Exp. 2................75

Figure 12. Group mean eta-squared $( \pm S E M)$ for the relative elapsed time scale and the absolute elapsed time scale for each group in Exp. 2...................76

Figure 13. Group mean $\mathrm{p}(\mathrm{long})( \pm S E M)$ as a function of the relative duration (log scale) for

Phases 1 and 2 in Exp. 2. ..................................... 77 


\section{Introduction}

The study of temporal perception was influenced in the late 1970s by new discoveries in animal learning operant and Pavlovian preparations, including the avoidance paradigm, fixedinterval (FI) schedules, choice procedures, and autoshaping (Gibbon, 1977; Church, 2002). For example, Gibbon, Baldock, Locurto, Gold, and Terrace (1977) found that the rate of acquisition of a conditioned response (CR) in autoshaping was a function not only of the conditioned stimulus (CS)-unconditioned stimulus (US) interval but also of the ratio of CS-US interval to the inter-trial interval (ITI). That is, although the absolute durations of the CS-US intervals may differ, the rate of acquisition of a CR is similar as long as the ratio of the CS-US interval to the CS-ITI interval remains the same. Gibbon et al. found this invariance using CS-US intervals ranging from 8 to $32 \mathrm{~s}$ and ITIs ranging from 48 to $384 \mathrm{~s}$. This finding suggests that a ratio rule for interval comparisons governs behavior. For FI schedules of reinforcement in instrumental conditioning, Dews (1970) observed the superposition of different FI functions (FI 30-s, FI 300s, and FI 3000-s) when relative rate was plotted on a normalized time axis, consistent with Weber's law for time (Gibbon, 1977). Behavior that follows a ratio rule and Weber's law for time is consistent with the scalar property, which is a central component of scalar expectancy theory (SET; Gibbon,1977). SET specifies that acquisition in autoshaping and response patterns in FI schedules are controlled by a proportional temporal distance in the inter-reinforcement interval rather than the absolute duration of the timed stimulus.

These discoveries that behaviors under Pavlovian and instrumental conditioning were, at least partly, controlled by a relative temporal aspect of the environment led to the development of theories of conditioning based on the properties of timing behavior such as SET (Gibbon, 1977), the behavioral theory of timing (Bet; Killeen \& Fetterman, 1988), the learning to time 
theory (LeT; Machado, 1997), and rate estimation theory (RET; Gallistel \& Gibbon, 2000), all of which account for behavior in many different conditioning paradigms. As a consequence, an interest in temporal perception per se re-emerged (Church, 2002). The studies of temporal perception asked questions such as " "What is the functional relationship between physical time and subjective time?' 'What is the relationship between temporal perception and temporal memory?' and 'What is the basis for a decision about the duration of a stimulus, or whether it was time to make a response?"' (Church, 2002, p. 269).

The temporal bisection procedure is a commonly used procedure aimed at studying temporal perception in non-human animals and humans (Church and Deluty, 1977; Stubbs, 1968; Wearden, 1991). This procedure requires a participant to make a different response following short- (S) and long- (L) duration standards (also called anchors). For example, an experimenter places a rat in an operant chamber, which is equipped with a houselight and two levers on the right and left sides of the front panel of the box. In discrimination training, the houselight is illuminated for a given duration (e.g., $2 \mathrm{~s}$ as $\mathrm{S}$ and $8 \mathrm{~s}$ as $\mathrm{L}$ ) and a response to one lever produces a food pellet according to the assignment of the duration and the location of the lever (e.g. given a 2-s houselight, a right lever press, and given a 8-s houselight, a left lever press). After discrimination training, subjects are exposed to a bisection test phase, in which trials consist of one of five intermediate, test durations in addition to the $\mathrm{S}$ and $\mathrm{L}$ anchor durations, where only correct responses to the anchor durations are reinforced. When the probability of a "long" response $(p[l o n g])$ in the presence of each of these seven timing stimuli is plotted as a function of stimulus duration, the resulting psychometric function resembles an ogival shape as in Figure 1. The bisection point (BP), which is one of the primary dependent variables of the procedure, is the stimulus duration corresponding to the probability of responding "long" on $50 \%$ of the trials, 
$\mathrm{p}($ long $)=.50$. The BP is also called the indifference point, or the point of subjective equality (PSE), between the anchor durations. Another measure of interest is gamma, $\gamma$, defined as the standard deviation of the BP divided by the BP. Gamma is the coefficient of variation that is proportional to the Weber fraction, which is the difference limen (DL) divided by the BP. DL is half the difference between stimulus durations corresponding to $\mathrm{p}(\mathrm{long})=.25$ and $\mathrm{p}(\mathrm{long})=.75$. Researchers use gamma and the Weber fraction interchangeably to measure underlying variability in timing behavior, and inverse measure of timing precision (Allan, 2002; Killeen, Fetterman, \& Bizo, 1997; Machado \& Keen, 2003). The BP and gamma indices of temporal perception are the primary foci of theoretical interpretations of timing behavior.

In a classic study, Church and Deluty (1977) exposed rats in the temporal bisection procedure to different sets of S and L durations including 1- vs. 4-s, 2- vs. 8-s, 3- vs. 12-s, and 4vs. 16-s. The authors reported three important findings. One was that the BP fell at the geometric mean (GM) of each set of anchor durations. Second, the DL increased as the values of the anchor sets increased. Third, the Weber fraction was constant across the anchor sets. The invariance of the Weber fraction, a measure of temporal sensitivity, across duration ranges supported Weber's law for temporal perception.

Gibbon (1981) theoretically explored the results of Church and Deluty (1977) in the framework of SET and suggested a specific ratio comparison rule to account for the performance in the temporal bisection procedure. The ratio similarity rule states that the $\mathrm{BP}$ is derived from a comparison of the sample stimulus duration to the internal representation of the short and long anchor durations. When the sample stimulus duration is closer to the representation of $\mathrm{S}$ than to that of $\mathrm{L}$, the subject responds 'short', and vice versa. For example, for $\mathrm{S}=2 \mathrm{~s}$ and $\mathrm{L}=8 \mathrm{~s}$, when a given test stimulus is $3 \mathrm{~s}$, the comparison with $\mathrm{S}$ yields $2 / 3=0.666$ and the comparison with $\mathrm{L}$ is 
$3 / 8=0.375$. Since $0.666>0.375$, the 3 -s duration will occasion a 'short' response. Formally stated, $\mathrm{S} / \mathrm{T}=\beta(\mathrm{T} / \mathrm{L})$, where $\mathrm{T}$ is a given test duration and $\beta$ is a bias term. If $\beta=1$, the unbiased $\mathrm{BP}=\sqrt{ } \mathrm{SL}$, which gives the $\mathrm{BP}$ at the GM of $\mathrm{S}$ and $\mathrm{L}$. The ratio similarity rule hypothesizes the BP at the GM, which was compatible with Church and Deluty's (1977) findings. Thus, the ratio similarity rule implies that both the short and long anchor durations influence the location of the $\mathrm{BP}$ in the temporal bisection procedure. A number of studies have found the $\mathrm{BP}$ at the GM of two trained durations in non-human animal research (e.g. Church \& Deluty, 1977; Meck, 1983; Meck \& Church, 1983; Stubbs, 1976), supporting the prediction of the ratio similarity rule.

The investigation of the nature of the $\mathrm{BP}$ has an important theoretical implication, as it is sometimes conceptualized as the midpoint between two alternative durations or as being based on a decision criterion. A subjective midpoint between two stimuli of the same dimension or two response options (choice behavior) is often a subject of investigation across different paradigms. For example, in the concurrent-chain paradigm, Fantino (1967) found that the midpoint of response allocation or indifference between a fixed-ratio (FR) schedule of reinforcement and a mixed-ratio schedule of reinforcement is found when the value of FR schedule and the GM of each reinforcement schedule component of the mixed-ratio schedule are equal. In the concurrent FI FI schedules of reinforcement paradigm, Platt and Davis (1983) investigated the point at which pigeons switched from one schedule to another. That is, they were interested in finding the BP-equivalent to the temporal bisection procedure in concurrent FI FI schedules. Pigeons were exposed to various concurrent schedules, where two keylights signaled different FI schedules of reinforcement. The conditions were (1) FI 40-s vs. FI 200-s, (2) FI 40-s vs. FI 120-s, (3) FI 40-s vs. FI 80-s, (4) FI 40-s vs. FI 200-s, (5) FI 60-s vs. FI 120-s, (6) FI 40-s vs. FI 60-s, (7) FI 60-s vs. FI 200-s, and (8) FI 100-s vs. FI 200-s (Exp. 1). They examined the response pattern during 
the FI intervals of each component and the probability of switching from one keylight to the other. They found that response rate on the short FI keylight increased as the short FI value approached, and then decreased after the short FI value passed, and there was an increase in response rate on the long FI keylight as the long FI value approached. More importantly, the point at which the response rate functions of the short and long FI schedule components crossed was at the GM of the two FI values. Also, the median switch time from the short FI keylight to the long FI keylight corresponded to the GM of the two FI values. They indicated that the subjective midpoint of two FI schedule values generally lay at the GM, similar to the findings in the temporal bisection procedure (e.g. Church \& Deluty, 1977), supporting the ratio similarity rule.

An exception in Platt and Davis (1983; Exp. 1) was with the FI 40-s vs. FI 200-s condition, where response rate after the short FI value passed reached a near-zero level, thus the crossing point was not calculable. This trend was also observed in Exp. 2, where they used long FI values such as FI 10-s vs. FI 160-s and FI 20-s vs. FI 120-s among other duration pairs. Platt and Davis suggested the ratio of two FI durations must be less than $4: 1$ for the crossing point to be at the GM of both FI values. They proposed that when the durations compared had a large difference $(>4: 1)$, the comparison between the two FI values would not occur and that there would be no measurable midpoint. That is, each FI schedule would have independent control of behavior and there would be no comparison between the two durations.

Evidence in the temporal bisection task also shows that the BP is not always a function of two anchor durations. For example, Raslear $(1983 ; 1985)$ reported the effect of the distribution of test stimuli and the probability of reinforcement on the location of the BP. Raslear (1983) exposed rats to 0.1 - versus 10 -s anchor durations using two different distributions. When the test 
stimulus distribution was logarithmic $(0.21,1,2.19,4.68 \mathrm{~s})$, the BP was at $1.4 \mathrm{~s}$, whereas when it was arithmetic $(1.34,3.81,5.05,6.29,8.76 \mathrm{~s})$, the $\mathrm{BP}$ was at $2.53 \mathrm{~s}$. That is, when the test distribution was arithmetic, the BP was higher than when it was a logarithmic progression.

Aside from the effect of the test duration distribution, Raslear (1985) also found that the probability of reinforcement influenced the location of the BP. He manipulated the probability of reinforcement on trials with the anchor durations of $0.1 \mathrm{~s}$ and $10 \mathrm{~s}$. The probability of reinforcement for each anchor duration varied from (1) 1.0 for $\mathrm{S}$ and 0.5 for $\mathrm{L}$, (2) 1.0 for $\mathrm{S}$ and 0.9 for $\mathrm{L}$, (3) 0.9 for $\mathrm{S}$ and 0.9 for $\mathrm{L}$, (4) 0.8 for $\mathrm{S}$ and 1.0 for $\mathrm{L}$, and (5) 0.5 for S and 1.0 for $\mathrm{L}$. He observed an effect of reinforcement probability on the location of the BP as follows: $2.56 \mathrm{~s}$ for the condition $1,2.85 \mathrm{~s}$ for condition $2,2.68 \mathrm{~s}$ for condition $3,2.34 \mathrm{~s}$ for condition 4 , and 2.00 $\mathrm{s}$ for condition 5. In general, the BP was larger when the probability of reinforcement for the L anchor was smaller than that of the $\mathrm{S}$ anchor as in the sets 1 and 2, compared to those conditions in which the probability of reinforcement was larger for the L compared to the S anchor, as in sets 4 and 5.

Machado and Keen (2003) reported that the change in the long anchor duration alone did not affect the location of the BP. Working with pigeons, they used a long rectangular chamber in which a timing cue was located in the center of the box and two response keys were located on the side walls. Two microswitches were located under the floors of the long chamber, separating the chamber into two regions. They measured the $\mathrm{p}$ (long) on the bisection tests and the time the birds departed from the 'short' key region and moved to the 'long' key region (departure time) among other measures. In Exp. 1, four pigeons were exposed to a 3- vs. 12-s condition. The average BP from the psychometric function was at $4.7 \mathrm{~s}$, less than the GM of $6 \mathrm{~s}$. The departure time correlated well with the BP. In Exp. 2, pigeons were exposed to 3- vs. 9-s and then 9- vs. 
27-s conditions. In both cases, both the BP, measured during the bisection test, and the departure time fell at the GM. They noted that the BP in the 3- vs. 12-s condition in Exp. 1 and the 3- vs. 9$\mathrm{s}$ condition in Exp. 2 (which fell at $5.1 \mathrm{~s}$ ) did not differ as would be expected from the ratio similarity prediction. Based on these observations, they inferred that the location of the BP was only a function of the short duration. That is, the BP might not be a reflection of the comparison between two anchors as the ratio similarity rule suggested. Machado and Keen addressed this issue in Exp. 3.

In Exp. 3, pigeons were exposed to two conditions, 3- vs. 9-s and the 3- vs. 27-s, in which the order of conditions was counterbalanced across birds. The average departure times were $4.7 \mathrm{~s}$ in the 3-vs. 9-s condition and $5.6 \mathrm{~s}$ in the 3-vs. 27 -s condition, values that did not differ significantly. That is, the departure time approximated the GM in the 3-vs. 9-s condition, but was lower than the GM in the 3-vs. 27-s condition. In phase 2, the same pigeons were exposed to 9- vs. 27-s and 9- vs. 81-s conditions, with order counterbalanced across pigeons. The departure times were $16 \mathrm{~s}(\mathrm{GM}=15.6 \mathrm{~s})$ and $18 \mathrm{~s}(\mathrm{GM}=27 \mathrm{~s})$, respectively, again not significantly different from each other. Since there was no significant increase in the BP even though the value of the long anchor increased, this outcome indicated to the authors that the location of the departure time was determined by the value of the short duration, and not influenced by the values of the long duration. This account, which is referred to here as the Short/no short hypothesis, questioned the validity of the ratio similarity rule. Unlike the ratio similarity rule, the Short/no Short hypothesis does not suggest a comparison involving the long anchor duration in the determination of the BP.

It is important to note that a deviation in the location of the BP from the GM of anchors could also be predicted by Killeen et al.'s (1997) contemporary version of signal detection model 
for the temporal bisection procedure called the Pseudo-Logistic Model (PLM). PLM indicates that the BP is located at the point where the memory distributions of the short and long anchor stimuli cross, which is called E. Killeen et al., derived the following relation:

$$
\mathrm{E}=\mathrm{HM}(1+\mathrm{k}), \text { where } \mathrm{k}=\mathrm{w} \ln (\mathrm{L} / \mathrm{S}) / 2
$$

where $\mathrm{HM}$ is the harmonic mean of the anchor durations, $\mathrm{S}$ and $\mathrm{L}$ are the values of the short and long anchor durations respectively, and $\mathrm{w}$ is gamma, the coefficient of variation that is proportional to the Weber fraction. In PLM, the location of the BP is determined by the HM of two anchor durations, the L:S ratio and gamma. Machado and Keen (2003) noted that PLM provided good estimates of the obtained BPs in their study.

Machado and Keen's (2003) study differed from Church and Deluty (1977) in several ways, including the use of a long rectangular box, departure time as an analogue measurement of the BP, a change in the long anchor duration from one phase to the next while holding the short anchor duration constant, a large S/L ratio, and pigeons as subjects, any of which might be responsible for the discrepancies in results between the two studies. An objective of the present study was to replicate Machado and Keen's (2003) study in a standard operant chamber with pigeons. 


\section{Experiment 1}

Exp. 1 investigated the effect of the long anchor duration on the location of the BP in a standard operant chamber with pigeons. In each discrimination training phase, a center keylight was presented as a timing cue signaling a short or long anchor duration, and two side keys were subsequently illuminated as the comparison stimuli. Pigeons were trained to peck at one side keylight following a short duration and the other side keylight following a long duration. Once the discrimination accuracy reached a 95\% criterion, a bisection test was given for four sessions. After the bisection test, the value of the long anchor duration was changed in the next discrimination training phase, and another test was run after the discrimination criterion was met. Two groups of naïve pigeons were first exposed to the 3-vs. 9-s condition (Phase 1), then to the 3- vs. 27-s condition (Phase 2). These anchor values were the same as in Machado and Keen (2003; Exp. 3). Subsequently, in Phase 3, Group Exp was exposed to anchor durations of 3- vs. 13.5-s and Group Control was exposed to durations of 3- vs. 27-s. That is, for Group Exp, the long duration was changed (halved), whereas there was no change for Group Control. In Phase 4, both groups were re-exposed to the 3 - vs 27 -s condition. Table 1 shows the design of Experiment 1. The interest of the study was whether the different values of the long anchor duration in each phase influenced the location of the BP. 


\section{Method}

\section{Subjects}

Twelve naïve male adult silver king pigeons were maintained at $80 \%$ of their free-feeding weights. All the birds were housed individually in home cages with water available. A 12:12h light-dark cycle was maintained in the home cage room. Experimental sessions were conducted seven days a week during the light period of the cycle.

\section{Apparatus}

Five sound-proofed operant chambers $(37 \mathrm{~cm} \mathrm{H} \mathrm{x} 31 \mathrm{~cm} \mathrm{~W} \mathrm{x} 34 \mathrm{~cm} \mathrm{D;} \mathrm{BRS/LVE,} \mathrm{Inc,}$ Laurel, MD, USA, Model SEC-002) with aluminum interior walls and a stainless steel mesh floor were used. The houselight lamp (Sylvania, No. 1829, 28VDC, 0.07A) was located on the front panel $1.5 \mathrm{~cm}$ under the ceiling and $17 \mathrm{~cm}$ from the right wall. The metal cover of the houselight fixture reflected the light toward the ceiling. There were three response keylight apertures $(2.5 \mathrm{~cm})$ on the front panel $8 \mathrm{~cm}$ from the ceiling and $8.25 \mathrm{~cm}$ apart from each other. Lucite pecking keys (BRS/LVE, PPK-001) were mounted behind the three apertures. The center key could be lit with either red or blue lights from a light source attached behind it (IC-901, pattern 696). The side keys could be illuminated with a green light (BRS/LVE, TQL-015). A food hopper aperture $(5.5 \mathrm{~cm} \mathrm{Wx} 5 \mathrm{~cm} \mathrm{H})$ was located $6 \mathrm{~cm}$ below the center keylight. A food magazine (BRS/LVE, GFM-001) was behind the food hopper aperture and filled with Purina Pigeon Checkers, which the subjects were fed on a daily basis in the home cage. A photocell assembly was attached on the side walls of the food hopper aperture to permit detection of the subjects' hopper entries. A 28v lamp (\#1829) was built inside the hopper aperture and turned on when the food magazine was activated. MED-PC IV software based on a Windows operating system was used for programming and the operations of all devices were on 28VDC. 


\section{Experimental Design}

Pigeons were assigned to two groups ( $n=6$ per group). The independent variable was the value of the long anchor duration, manipulated across phases. The experimental group was exposed to the sequence: 3- vs. 9-s, 3-vs. 27-s, 3- vs. 13.5-s, and 3-vs 27-s in phases 1 to 4, respectively. The control group was exposed to the same sequence except for phase 3 in which the anchors remained 3- vs 27-s. This design permitted a comparison of conditions among phases (phases 1, 2, and 4) as well as between groups (phase 3). Pigeons were randomly assigned to each group. They were then assigned to each of five operant chambers in a way that at least one bird from each group was assigned to one of the five boxes. Experimental sessions were about an hour and half in duration and were run in successive squads of 2-5 pigeons. There was a total of five squads a day and the squad assignment was partially balanced between the groups in a way that at least one bird from each group was assigned to each squad. The assignment of the comparison-key designation (short vs. long) and locations (left vs. right) was counterbalanced across subjects within a group.

\section{Procedure}

Magazine training. Pigeons were randomly assigned to either Group Exp $(n=6)$ or Group Control $(n=6)$. All subjects were trained to eat from the food hopper. The food hopper was always illuminated when it was activated. The houselight was illuminated throughout the session. In the first session of magazine training, the experimenter overfilled the already activated food hopper on the first trial, which began as the experimenter placed the subject into the chamber. When the subject inserted its head into the hopper, the hopper retracted after $40 \mathrm{~s}$. Thirty seconds later, the hopper activated again and continued this cycle for 20 trials, during which the duration of the access to food decreased on each trial from $40 \mathrm{~s}$ to $3 \mathrm{~s}$ in the order $40 \mathrm{~s}$, 
$30 \mathrm{~s}, 10 \mathrm{~s}, 5 \mathrm{~s}$, and then $3 \mathrm{~s}$, for the remaining 16 trials, timed from the detection of the bird's head in the food magazine aperture. There was no latency requirement for the head entry. Magazine training ended when a pigeon successfully ate from the hopper on more than $90 \%$ of the trials for a session. If a pigeon did not make the head entry responses more than $90 \%$ within $30 \mathrm{~min}$, the session was terminated and another session was given the next day.

Observing key training. An autoshaping procedure was used to train pecking to the red center observing key. A trial began with the presentation of the red center keylight. A peck to the center keylight or a passage of $5 \mathrm{~s}$ without pecking terminated the center keylight and produced a 3 -s access to food. The inter-trial interval was an average of $30 \mathrm{~s}(20,30$, or $40 \mathrm{~s})$. There were 60 trials per session. If the subject pecked the center key on more than 30 trials within a session, the subject moved on to the next (side-key training) phase. All subjects met the criterion within three sessions.

Side-key training. A trial began with the presentation of the red center keylight. A peck to the center keylight turned off the center keylight and turned on either right or left keylight illuminated in green. Pecking to the side keylight or a passage of $5 \mathrm{~s}$ terminated the side key and produced a 2-s presentation of food. There were 60 trials per session with 30 presentations of the right and left keys each. The order of the side keylight presentations was randomized in blocks of six trials. The criterion for moving to the next (discrimination training) phase was a peck to the sidekey on more than 30 trials within a session.

Discrimination training: 100\% Forced choice. The anchor durations of the timing key were $3 \mathrm{~s}$ and $9 \mathrm{~s}$. A session started with the onset of the red center keylight. A peck to the red keylight turned the color to blue. The blue center keylight was the sample cue; it lasted for a given anchor duration and its termination coincided with the presentation of one comparison side 
key (left or right), associated with the correct response for that anchor duration. A peck to the comparison side key produced the 2-s access to food and terminated the comparison keylight, ending the trial, and initiating an ITI, which average duration was $30 \mathrm{~s}$ as in previous phases. A peck to a dark side key had no programmed consequences. A failure to peck the observing key for $10 \mathrm{~s}$ resulted in the termination of the trial. The comparison side key was presented until the pigeon pecked it. The assignment of the anchor duration and the location of a correct response on the comparison keys was counterbalanced across subjects. There were 80 trials per session (40 trials for each anchor duration). This phase lasted for three sessions.

Discrimination training: 50\% Forced choice. This phase was identical to the 100\% Forced choice phase except for the following. Half the trials of a session (40 trials) were freechoice trials, with an equal number of trials for each anchor duration. The free-choice trial was different from the forced choice trial in that the termination of the timing cue coincided with the presentation of both comparison side keys. A correct response produced access to food. Pecking the incorrect key produced no access to food and terminated the trial. Not pecking either key for $10 \mathrm{~s}$ led to the termination of the trial and no food delivery. This phase lasted for three sessions.

Discrimination training: 0\% Forced choice. The session consisted of only free-choice trials. There were 80 trials per session (40 trials for each anchor duration). A mastery criterion to move to the next phase for each pigeon was $95 \%$ correct responses, regardless of the duration, for one session. Each pigeon moved to the next test phase upon meeting the discrimination training criterion.

Phase 1: Bisection test. The session was identical to the previous phase except for the following. In addition to the 3 and $9 \mathrm{~s}$ anchor durations, intermediate durations of 3.6, 4.3, 5.2, 6.2 , and $7.5 \mathrm{~s}$ were presented. The test duration distribution was geometrically spaced between 
the short duration and the long duration as in Church and Deluty (1977). Correct responding to 3 and $9 \mathrm{~s}$ durations produced access to food, whereas responding during the other test duration trials ended without food presentation. There were 90 trials per session. The anchor durations were presented on 30 trials each. Each unreinforced test duration was presented for 6 trials. The order of the trial presentations was random. There were four sessions.

Phase 2: discrimination training. This phase was the same as Discrimination training: $0 \%$ Forced choice except that the anchor durations were 3 and 27 s. A minimum of five sessions and the mastery criterion (95\% correct) were required to move to the next test phase.

Phase 2: Bisection test. This session was identical to the previous Phase 1: Bisection test except for the following: The anchor durations were 3 and $27 \mathrm{~s}$ and the test durations were 4.3, $6.2,9,13$, and $18.7 \mathrm{~s}$. There were four sessions.

Phase 3: discrimination training. For Group Exp, the anchor durations of the timing key were $3 \mathrm{~s}$ and $13.5 \mathrm{~s}$, whereas for Group Control, the anchor durations were 3 and $27 \mathrm{~s} . \mathrm{A}$ minimum of five sessions and the mastery criterion (95\% correct) were required to move to the next test phase.

Phase 3: Bisection test. This session was identical to the previous Phase 1: Bisection test except for the following: For Group Exp, the anchor durations were 3 and $13.5 \mathrm{~s}$ and the test durations were 3.8, 4.9, 6.3, 8.1, and 10.4 s. For Group Control, the anchor durations were 3 and $27 \mathrm{~s}$ and the test durations were $4.3,6.2,9,13$, and $18.7 \mathrm{~s}$. There were four sessions.

Phase 4: discrimination training. For both groups, the anchor durations were 3 and $27 \mathrm{~s}$. A minimum of five sessions and the mastery criterion (95\% correct) were required to move to the next test phase. 
Phase 4: Bisection test. For both groups, the anchor durations were 3 and $27 \mathrm{~s}$ and the test durations were 4.3, 6.2, 9, 13, and $18.7 \mathrm{~s}$. There were four sessions.

\section{Data Analysis}

BP and gamma. The BP and gamma were estimated from the PLM (Killeen, Fetterman, \& Bizo, 1997) using GraphPad Prism 5 software (Brown, et al., 2011; Grommet, 2013). Each pigeon's average $\mathrm{p}$ (long) values from the four bisection test sessions in each phase was fitted into the PLM.

The PLM formula was based on Allan (2002; Eq. 3):

$$
P\left(R_{L}\right)=\left[1+\exp \left(T_{1 / 2}-t\right) /\left(\sqrt{ } 3 / \pi^{*} \sigma_{t}\right)\right]^{-1},
$$

where perceived time, $\sigma_{\mathrm{t}}=\sqrt{ }\left[(\gamma \mathrm{t})^{2}+p t+c^{2}\right]$, in which $\gamma$ is the constant of proportionality corresponding to scalar variability, $p$ is the constant of proportionality corresponding to Poisson variability, and $c$ is a constant error. The present study made the assumption that scalar variance, $\gamma$, dominates in the variance in $\sigma_{\mathrm{t}}$ and $p$ and $c$ were set to 0 , as did Allan (2002). Thus, the PLM formula of the present study became:

$$
\mathrm{P}\left(\mathrm{R}_{\mathrm{L}}\right)=\left[1+\exp \left(\mathrm{T}_{1 / 2}-\mathrm{t}\right) /\left(\sqrt{3} / \pi^{*} \gamma^{*} \mathrm{t}\right)\right]^{-1},
$$

where $\gamma$, gamma, replaces $\sigma_{\mathrm{t}}$ (Allan, 2002; Eq. 5). In this equation, $\mathrm{T}_{1 / 2}$ is the $\mathrm{BP}$ and gamma is proportional to the Weber ratio. The derived BPs and gammas were used as a primary dependent variable of this study.

The goodness of fit of the psychometric function by the PLM was calculated for each pigeon as the average proportion of variance accounted for, and the average goodness of fit across groups and phases yielded as $r^{2}=.97$, showing an overall good fit by the model.

Statistical Analysis. All statistical analyses used an $\alpha$ level of .05. 


\section{Results}

\section{Bisection point}

The BP for each pigeon was derived from the individual psychometric functions using equation 3. Figure 2 shows the group mean BP as a function of the phases for two groups in Exp. 1. For Group Exp, the BP was $5.29 \mathrm{~s}(S E M=.17)$ in the 3 - vs. 9-s condition, $7.78 \mathrm{~s}(S E M=1.11)$ in the 3- vs. 27-s condition, $5.88 \mathrm{~s}(S E M=.56)$ in the 3 - vs. 13.5-s condition, and $7.6 \mathrm{~s}(S E M=$ $1.21)$ in the 3- vs. 27-s condition. For Group Control, the BP was $5.17 \mathrm{~s}(S E M=.22)$ in the 3-vs. 9-s condition, $7.25 \mathrm{~s}(S E M=.60)$ in the 3 - vs. 27-s condition, 8.01 s $(S E M=.90)$ in the 3-vs. 13.5-s condition, and $7.97 \mathrm{~s}(S E M=.90)$ in the 3 - vs. 27 -s condition.

To assess whether there was an effect of a long anchor value across phases, a Group x Phase analysis of variance (ANOVA) of the BP was conducted. The analysis yielded a main effect of Phase, no effect of Group, and no Group x Phase interaction (Table 2). Since there was no group differences, data from two groups were pooled and one-way ANOVA of the BP was conducted. The analysis indicated a significant difference across phases, $F(3,33)=6.41, p<$ 0.01. Tukey HSD test indicated that the BP was significantly different between Phases 1 and 2 and Phases 1 and 4, but not elsewhere. Importantly, the BP significantly increased from the 3-vs 9- condition (Phase 1) to the 3- vs 27-s condition (Phase 2), which was not observed in Machado and Keen (2003; Exp. 3). When the analysis was restricted to Phases 2 and 3, a Group x Phase ANOVA of the BP yielded no effect of Group, $F(1,10)=0.55, p=0.48$, or no effect of Phase, $F(1,10)=1.86, p=0.20$, but there was a Group $\mathrm{x}$ Phase interaction, $F(1,10)=10.12, p<0.01$. The interaction indicated that the difference in the BP from the 3- vs. 27-s condition to the 3-vs. 13.5-s condition for Group Exp was greater than when the 3-vs. 27-s condition remained constant across phases 2 and 3 for Group Control. The simple main effect of Phase was tested for 
both groups. For Group Exp, there was a significant difference between phases, $t(5)=2.65, p<$ 0.05, whereas, for Group Control, there was no difference between phases, $t(5)=-1.75, p=0.14$, showing that the change in the long anchor duration influenced the location of the BP.

The locations of the BP in each phase for each group were assessed for their approximations to the GM of the two anchor duration as predicted by the ratio similarity rule. For Group Exp, the BP was not significantly different from the GM in conditions 3- vs. 9-s (Phase 1), $t(5)=0.54, p=0.61,3$ - vs. 27-s (Phase 2), $t(5)=-1.11, p=0.32,3$ - vs. 13.5-s (Phase $3), t(5)=-0.93, p=0.39$, or 3 - vs. 27-s (Phase 4), $t(5)=-1.16, p=0.3$. Thus, for Group Exp, the value of the long anchor duration influenced the location of the BP for all the conditions as predicted by the ratio similarity rule. For Group Control, the BP was not significantly different from the GM in conditions 3- vs. 9-s (Phase 1), $t(5)=-0.14, p=0.89,3$ - vs. 27-s (Phase 3), $t(5)$ $=-1.09, p=0.32$, and 3- vs. 27-s (Phase 4), $t(5)=-1.13, p=0.31$, but there was a difference in condition 3- vs. 27-s (Phase 2), $t(5)=-2.91, p=0.03$. For Group Control, the BP fell at the GM of the two anchor durations in all the phases except in Phase 2. These results indicated that both the short and long anchor durations influenced the location of the BP across different phases and they were mostly in accordance with the prediction of the ratio similarity rule.

\section{Sample-cue responding}

Interestingly, pigeons pecked at the timing cue during the presentation of the short or long anchor durations even though there were no programmed consequences for such responding. This behavior can be referred to as sample-cue responding. Figure 3 shows the response rate (responses per second) on the sample cue as a function of elapsed time on long anchor stimulus trials, averaged across two groups for the first two phases. These data were taken from the last sessions of discrimination training in the 3- vs. 9-s condition in Phase 1 and 
the 3- vs. 27-s condition in Phase 2, where each pigeon met the mastery criterion. As can be seen in Figure 3, sample-cue responding showed a V-shape pattern for which response rate was initially high, followed by a decrease, then another increase toward the end of the sample duration. This V-shape pattern was evident for both the 3-vs. 9-s condition and the 3-vs. 27-s condition, suggesting that the sample-cue duration exerted temporal control over the pattern of the sample keypeck response in both phases. 


\section{Discussion}

Experiment 1 investigated the effect of the long duration on the location of the BP. Two groups of naïve pigeons were first exposed to the 3- vs. 9-s condition (Phase 1), then to the 3- vs. 27-s condition (Phase 2). Subsequently, Group Exp was exposed to anchor durations of 3- vs. 13.5-s and Group Control was exposed to durations of 3- vs. 27-s in Phase 3. Both groups were re-exposed to the 3- vs 27-s condition (Phase 4). Exp. 1 found that there was a significant increase in the BPs from the 3- vs 9- condition (Phase 1) to the 3-vs 27-s condition (Phase 2). Thus, even though the values of the short and long anchors were the same as in Machado and Keen (2003; Exp. 3), Exp. 1 revealed an effect of the long anchor value on the location of the BP. Moreover, for Group Exp, the BP was at the GM of two anchor durations in each phase. These results were consistent with the prediction of the ratio similarity rule.

Sample-cue responding was observed during the presentation of the timing cue. The response functions showed a $\mathrm{V}$-shape pattern where response rate was initially high, followed by a decrease, then another increase toward the end of the sample duration. This V-shape pattern was evident for both the 3- vs. 9-s condition (Phase 1) and the 3- vs. 27-s condition (Phase 2), suggesting that elapsed stimulus duration exerted temporal control over the pattern of the key peck response.

Both the results of the BP and sample-cue responding suggested that performance in the temporal bisection procedure was influenced by both the short and long durations in the standard operant chamber. This observation was different from that of Machado and Keen (2003; Exp. 3) who observed no change in the departure time when they moved the value of the long anchor duration from $9 \mathrm{~s}$ to $27 \mathrm{~s}$ or from $27 \mathrm{~s}$ to $9 \mathrm{~s}$ in their long box. This difference might be due to the use of the long operant chamber as opposed to the standard operant chamber. Exp. 1 did not 
control for a possible order effect and did not conclusively refute Machado and Keen's (2003)

findings that the BP did not change when there was a change in the long anchor value only.

Experiment 2 was conducted to further explore the role of the long anchor duration, as in

Experiment 1, with the order of conditions counterbalanced. 


\section{Experiment 2}

The results of Experiment 1, conducted in a standard operant chamber, suggested that the $\mathrm{BP}$ was a function of both the short and long anchor stimuli. Experiment 2 was designed to control for order of treatments with two groups: Group 3-9 to 3-27 was exposed to the 3-vs 9-s condition followed by the 3-vs. 27-s condition in Phase 1 and Phase 2, respectively, whereas Group 3-27 to 3-9 was exposed to the 3-vs 27-s condition in Phase 1 and the 3- vs 9-s condition

in Phase 2. Thus, a systematic replication of Machado and Keen's (2003) study with pigeons in a standard operant chamber with pigeons was conducted. 


\section{Method}

\section{Subjects}

Twenty naïve male adult silver king pigeons were maintained at $80 \%$ of their freefeeding weights. The treatment of the birds was the same as in Experiment 1.

\section{Apparatus}

The apparatus was identical to that in Experiment 1.

\section{Experimental Design}

A balanced crossover design was used. Pigeons were assigned to two groups $(n=10$ per group). The within-subject independent variable was the value of the long anchor duration (9 vs. $27 \mathrm{~s}$ ) of the temporal bisection conditions across two phases. The between-subject variable was order of presentation of the two long-duration values. Table 3 shows the scheme of Experiment 2. The box assignment and the squad assignment were counterbalanced between groups, and the assignment of the comparison keys to locations was counterbalanced across subjects within a group.

\section{Procedure}

The following initial phases of the procedure were the same as in Experiment 1:

Magazine training, Observing key training, and Side-key training.

Discrimination training: 100\% Forced choice. Pigeons were randomly assigned to either Group 3-9 to 3-27 or Group 3-27 to 3-9. For Group 3-9 to 3-27, the initial anchor durations of the timing key were $3 \mathrm{~s}$ and $9 \mathrm{~s}$, whereas for Group 3-27 to 3-9, the initial anchor durations were 3 and 27 s. A session started with the onset of the red center keylight. A peck to the red keylight turned the color to blue. The blue center keylight was the sample cue; it lasted for a given anchor duration and its termination coincided with the presentation of one comparison side key (left or 
right), associated with the correct response for that anchor duration. A peck to the comparison side key produced 2-s access to food and terminated the comparison keylight, ending the trial, and initiating an ITI, which average duration was $30 \mathrm{~s}$ as in previous phases. A peck to a dark side key had no programmed consequences. A failure to peck the observing key for $10 \mathrm{~s}$ resulted in the termination of the trial without reinforcement. The comparison side key was presented until the pigeon pecked it. The assignment of the anchor duration and the location of a correct response on the comparison keys were counterbalanced across subjects within each group. There were 80 trials per session (40 trials for each anchor duration). This phase lasted for three sessions.

Discrimination training: 50\% Forced choice. This phase was identical to the $100 \%$ Forced choice phase except for the following. Half the trials of a session (40 trials) were freechoice trials, with an equal number of trials for each anchor duration. The free-choice trial was different from the forced choice trial in that the termination of the timing cue coincided with the presentation of both comparison side keys. A correct response produced access to food. Pecking the incorrect key produced no access to food and terminated the trial. Not pecking either key for $10 \mathrm{~s}$ led to the termination of the trial and no food delivery. This phase lasted for three sessions.

Discrimination training: 0\% Forced choice. The session consisted of only free-choice trials. There were 80 trials per session (40 trials for each anchor duration). A mastery criterion to move to the next phase for each pigeon was $95 \%$ correct responses, regardless of the duration, for one session. Each pigeon moved to the next test phase upon meeting the discrimination training criterion.

Phase 1: Bisection test. The session was identical to the previous phase except for the following. For Group 3-9 to 3-27, in addition to the 3 and 9 s anchor durations, intermediate 
durations of 3.6, 4.3, 5.2, 6.2, and 7.5 s were presented. For Group 3-27 to 3-9, the anchor durations were 3 and $27 \mathrm{~s}$ and the test durations were $4.3,6.2,9,13$, and $18.7 \mathrm{~s}$. The test duration distribution was geometrically spaced between the short duration and the long duration as in Church and Deluty (1977). Correct responding on comparison keys on trials with anchor durations produced access to food, whereas responding on trials with the other test durations ended without food presentation. There were 90 trials per session. The anchor durations were presented on 30 trials each. Each unreinforced test duration was presented for 6 trials. The order of the trial presentations was random. There were four sessions.

Phase 2: discrimination training. This phase was the same as Discrimination training: 0\% Forced choice except that the anchor durations for Group 3-9 to 3-27 were 3 and 27 s and for Group 3-27 to 3-9 were 3 and 9 s. A minimum of five sessions and the mastery criterion (95\% correct) were required to move to the next test phase.

Phase 2: Bisection test. This session was identical to the previous Phase 1: Bisection test except for the following: For Group 3-9 to 3-27, the anchor durations were 3 and $27 \mathrm{~s}$ and the test durations were 4.3, 6.2, 9, 13, and 18.7 s. For Group 3-27 to 3-9, the anchor durations were 3 and $9 \mathrm{~s}, 3.6,4.3,5.2,6.2$, and $7.5 \mathrm{~s}$. There were four sessions.

\section{Data Analysis}

Psychometric function. A psychometric function for each subject was derived from the average $\mathrm{p}$ (long) responses to each of seven duration stimuli in a bisection test of each phase. Each subject's p(long) data point was calculated as the number of "long" responses divided by the sum of the "long" responses and the "short" responses for each stimulus across four sessions. There were four test sessions in each phase. The average psychometric function was the average $\mathrm{p}$ (long) function of a group of pigeons. 
BP and gamma. The BP and gamma were estimated from equation 3 as in Experiment 1. The average goodness of fit across groups and phases yielded as $r^{2}=.95$, showing an overall good fit by the PLM model.

Statistical Analysis. All statistical analyses used an $\alpha$ level of .05. 


\section{Results}

\section{Discrimination training}

The number of sessions to acquisition in the 3- vs. 9-s condition and in the 3-vs. 27-s condition between Group 3-9 to 3-27 and Group 3-27 to 3-9 was assessed. Figure 4 shows the mean number of sessions to mastery criterion as a function of phases for Group 3-9 to 3-27 and Group 3-27 to 3-9. A Group (2) x Phase (2) ANOVA of the number of sessions to acquisition showed a main effect of Group, $F(1,18)=6.52, p=0.02$, a main effect of Phase, $F(1,18)=$ $27.18, p<0.01$, and a significant interaction of Group x Phase, $F(1,18)=20.70, p<0.01$. That is, the difference in the number of sessions to acquisition between two phases depended on whether or not the phases began with the 3-vs. 9-s condition or with the 3-vs. 27-s condition.

The simple main effect of group was tested for each phase. The comparison showed that there was a significant difference in the mean sessions to acquisition between the two groups in phase $1, t(9)=5.19, p=0.02$, and in phase $2, t(9)=6.90, p<0.01$. Group 3-9 to 3-27 took more sessions to reach acquisition than did Group 3-27 to 3-9 in Phase 1, but this difference was reversed in Phase 2.

The simple main effect of phase was tested for each group. For Group 3-9 to 3-27, mean sessions to acquisition was significantly greater in Phase 1 than in Phase $2, t(9)=6.16, p<0.01$. For Group 3-27 to 3-9, the mean sessions to acquisition did not differ significantly between phases, $t(9)=0.54, p=0.59$. Thus, more sessions were needed to reach to the mastery criterion for the 3- vs. 9-s condition than for the 3- vs. 27-s condition for Group 3-9 to 3-27, while there was no evidence of a difference in sessions to acquisition for Group 3-27 to 3-9. In both phases acquisition was faster under a 3-vs. 27-s condition than under a 3-vs. 9-s condition, but the difference was more pronounced in Phase 1 than in Phase 2. 


\section{Psychometric function}

The psychometric function was obtained from each individual subject's p(long) responses pooled across the four test sessions. Figure 5 shows the group mean probability of "Long" responding, $\mathrm{p}$ (long), as a function of stimulus duration for phases 1 and 2 for Group 3-9 to 3-27 (upper panel) and Group 3-27 to 3-9 (lower panel). For all phases for both groups, p(long) increased as the stimulus duration increased.

\section{Bisection point}

The BP for each pigeon was derived from the individual psychometric functions using equation 3 and is shown in Figure 6, which plots group mean BP as a function of phase for each group. The group mean BPs were compared between conditions 3- vs. 9-s and 3- vs. 27-s. For Group 3-9 to 3-27, the BP was $5.22 \mathrm{~s}(S E M=.13)$ and $8.73 \mathrm{~s}(S E M=.57)$ in the 3- vs. 9-s and the 3- vs. 27-s conditions, respectively. For Group 3-27 to 3-9, the BP was $4.96 \mathrm{~s}$ (SEM=.17) and $6.24 \mathrm{~s}(S E M=.32)$ in the 3- vs. 9-s condition and the 3-vs. 27-s condition, respectively. A Group (2) x Long (2) ANOVA of BP showed a main effect of Group, $F(1,18)=15.90, p<0.01$, a main effect of Long, $F(1,1)=47.18, p<0.01$, and a significant Group x Long interaction, $F(1$, $18)=10.29, p<0.01$. The main effect of Long indicated that the BPs in the 3-vs. 9-s condition and the 3-vs. 27-s condition were significantly different, showing that the value of the long anchor duration influenced the location of the BP, unlike the results of Machado and Keen (2003). On the other hand, the Group x Long interaction showed that the degree of difference in BP between two conditions differed between the groups.

The simple main effect of Long on BP was significant for both Group 3-9 to 3-27, $t(9)=$ 5.64, $p<0.01$, and Group 3-27 to 3-9, $t(9)=4.06, p<0.01$. That is, for both groups, the BP was greater under condition 3- vs. 27-s than under condition 3-vs 9-s. On the other hand, the simple 
main effect of Group was significant for the 3- vs. 27-s condition, $t(18)=3.79, p<0.01$, but not for the 3-vs. 9-s condition, $t(18)=1.20, p=0.24$. This pattern of results suggests an order effect, as the BP under condition 3- vs. 27-s was greater when it followed condition 3- vs. 9-s (Group 3-9 to 3-27) that when it was the initial condition (Group 3-27 to 3-9).

The locations of the BP in each phase for each group were assessed for their approximations to the GM of the two anchor duration as predicted by the ratio similarity rule as in Table 4. In the 3- vs 9-s condition, the BPs for both groups were not significantly different from the GM (5.2 s) of the two anchor durations, $t(9)=0.167, p=0.87$ and $t(9)=1.392, p=0.19$ for Group 3-9 to 3-27 and Group 3-27 to 3-9, respectively. The BPs for both groups were significantly greater than the HM (4.5 s) of the two anchor durations, $t(9)=5.45, p<0.01$ and $t(9)=2.70, p=0.03$ for Group 3-9 to 3-27 and Group 3-27 to 3-9, respectively. In the 3 vs 27 condition, the BP for Group 3-9 to 3-27 was not significantly different from the GM (9s), $t(9)=$ $0.472, p=0.65$, and was greater than the $\operatorname{HM}(5.4 \mathrm{~s}), t(9)=5.81, p<0.01$, whereas that for Group 3-27 to 3-9 was significantly lower than the GM, $t(9)=8.53, p<0.01$ and higher than the $\mathrm{HM}(5.4 \mathrm{~s}), t(9)=2.58, p=0.03$. This result is consistent with the observation that the BP in the 3- vs. 27-s condition varied depending on whether it was the initial condition or followed the 3vs. 9-s condition. Considering groups separately, for Group 3-9 to 3-27, the BPs in both conditions approximated the GM of the two anchor durations, consistent with the ratio similarity rule. For Group 3-27 to 3-9, the difference in the value of BPs across two conditions was small, similar to the findings of Machado and Keen (2003).

\section{Gamma}

In addition to the estimate of BP, PLM fit provided an estimate of gamma. Figure 7 shows group mean gamma as a function of phase for Group 3-9 to 3-27 and Group 3-27 to 3-9. A 
Group (2) x Long (2) ANOVA of gamma showed a main effect of Group, $F(1,18)=7.20, p=$ 0.02, a main effect of Long, $F(1,18)=8.87, p<0.01$, but no significant Group x Long interaction, $F(1,18)=2.31, p=0.15$. The value of gamma was significantly smaller in the 3 - vs. 9-s condition compared to in the 3-vs. 27-s condition. However, unlike the BP, the effect of the order was not detected in gamma.

\section{PLM prediction}

Both the BP and gamma varied as a function of the long anchor duration, in line with the relation predicted by PLM. Figure 8 shows the BPs of the individual subjects $(N=20)$ for both phases plotted against PLM prediction based on equation 1. The diagonal line represents the line for no difference between the two measures. The correlation between these measures was $r(18)=$ $.73, p<0.01$, indicating that PLM could account for some of the variation $\left(r^{2}=.53\right)$ of the present study's results. Machado and Keen (2003) also observed that there was a close match between the group average BPs of each condition of all the experiments $(N=5)$ with the PLM predictions (p. 179).

\section{Sample-cue responding}

As in Experiment 1, the pecking responses to the timing cue before the presentation of the comparison keys were observed during discrimination training and testing sessions. In the present study, data from the discrimination training session in which each subject met the mastery criterion for each phase were used for this analysis, as the data from testing were more variable. Figure 9 shows response rate (responses per second) during the sample cue on the long anchor trials plotted as a function of elapsed time in a trial in the two phases for both groups. For Group 3-9 to 3-27, response rate in both phases showed a V-shape pattern decreasing initially and increasing toward the end of the trial. For Group 3-27 to 3-9, response rate was high in the 
first few seconds and then decreased sharply to lower levels afterwards in both phases. It seemed that the sample-cue response pattern that developed in the first phase of the experiment differed between groups and carried over to the second phase of the experiment, as the shapes of the functions looked similar within groups but different between the groups.

In order to evaluate this visual impression, two separate analyses were performed based on different scales for the abscissa. The first analysis compared the shapes of the two functions across phases in each group when plotted in absolute elapsed trial time. Figure 9 suggests that for the first $9 \mathrm{~s}$ of the trial that was in common for all functions, the two functions for Group 3-9 to 3-27 look different from each other in shape on the elapsed time scale, whereas the two functions for Group 3-27 to 3-9 looked similar to each other. In order to conduct a quantitative analysis of the response rate pattern corrected for overall level of responding, the response rate for each pigeon was first normalized by taking the rate in each bin of a response function as a proportion of the minimum rate and the maximum rate of the function as:

$$
\text { Normalized rate }=(\text { rate }- \text { minimum rate }) /(\text { maximum rate }- \text { minimum rate })
$$

After each pigeon's sample-cue response function was normalized, the average sample-cue responding for each group in each phase was derived. Figure 10 shows the group mean normalized response rate as a function of elapsed time during the first $9 \mathrm{~s}$ of the long anchor trial for both phases (the 3- vs. 9-s condition and the 3-vs. 27-s condition) for both groups. A mixed Group (2) x Phase (2) x Time (9) ANOVA of normalized rate was conducted, and the results can be found in Table 5. In addition to a main effect of Time and two-way interactions, Group x Time and Phase x Time, the analysis yielded a Group x Phase x Time interaction. The three way interaction of Group $\mathrm{x}$ Phase $\mathrm{x}$ Time indicated that the relation of the temporal pattern of normalized rate to phase differed between groups. 
To parse the 3-way interaction, a Phase (2) x Time (2) ANOVA of normalized rate was conducted for each group. The analysis for Group 3-9 to 3-27 yielded no main effects of Phase or Time, but the Phase $\mathrm{x}$ Time interaction was significant (Table 6). That is, the temporal pattern of normalized response rate differed between phases for Group 3-9 to 3-27, with higher rate in phase 2 than in phase 1 early in the trial, and a reversal to lower rates in phase 2 later in the trial. For Group 3-27 to 3-9, a Phase (2) x Time (2) ANOVA of the normalized rate showed no main effect of Phase, but the main effect of Time and the interaction of Phase $x$ Time (Table 7). That is, the pattern of normalized response rate as a function of elapsed time for this group also depended on phase although patterns were similar across phases with no reversals of rate. The strength of the interaction for both groups were assessed by calculating the effect size for Phase $\mathrm{x}$ Time interaction (partial eta-squared $=$ SS for Phase $\mathrm{x}$ Time interaction divided by the sum of SS for Phase $\mathrm{x}$ Time interaction and SS for the Subject x Phase $\mathrm{x}$ Time error term). For Group 3-9 to 3-27, the effect size of the Phase $x$ Time interaction was $\eta^{2}=0.66$, whereas, for Group 3-27 to 39, the effect size was $\eta^{2}=0.19$. Thus, even though both Group 3-9 to 3-27 and Group 3-27 to 3-9 showed significant interaction, the effect size comparison suggested that the functions for Group 3-27 to 3-9 overlapped more than those for Group 3-9 to 3-27, supporting the visual impression in Figure 10. In sum, on the absolute-time scale, sample-cue response pattern superposed better for Group 3-27 to 3-9 compared to these of Group 3-9 to 3-27.

When elapsed time was plotted on an absolute scale, the functions of two conditions were more similar for Group 3-27 to 3-9 than for Group 3-9 to 3-27. This outcome indicates that the sample-cue response patterns in both phases were controlled in similar ways by absolute elapsed time for Group 3-27 to 3-9, but not for Group 3-9 to 3-27. An alternative account of the response patterns for Group 3-9 to 3-27 is that it varied with relative time, in conformity with the scalar 
property, as suggested by the similar V-shaped patterns for Group 3-9 to 3-27 in Figure 9. In order to evaluate this possibility, the data were re-plotted in relative elapsed time. The elapsed time scale for the 3- vs. 27-s condition of both groups was normalized with that of the 3- vs. 9-s condition by taking the average response rate in blocks of $3 \mathrm{~s}$, producing nine data points in total. Response rate was converted to a proportion for subjects in both groups based on the same operation as above (equation 4). If the functions across phases were similar in relative time, it would imply scalar timing of the sample duration.

Figure 11 shows group mean normalized response rate as a function of relative elapsed time on long anchor trials for both the 3-vs. 9-s condition and the 3-vs. 27-s condition for both groups. The results of a mixed Group (2) x Phase (2) x Time (9) ANOVA of normalized samplecue response rate can be found in Table 8. The analysis yielded main effects of Group, Time, and Phase, as well as two-way interactions, Group x Time, and Phase x Time interaction. In addition the Group x Phase $\mathrm{x}$ Time interaction was significant. The latter interaction indicated that the relation of the temporal pattern of normalized rate to phase differed between groups.

For Group 3-9 to 3-27, a Phase (2) x Time (2) ANOVA of normalized rate showed no main effect of Phase, the main effect of Time, and no Phase x Time interaction (Table 9). This result indicated that the normalized response rate changed as a function of normalized elapsed time, but there was no evidence of a difference in pattern between the two functions, giving support to the visual impression that the shapes of two functions were similar. This outcome implies conformity with the scalar property in the temporal control of behavior by the samplecue duration for Group 3-9 to 3-27.

For Group 3-27 to 3-9, a Phase (2) x Time (2) ANOVA of normalized sample-cue response rate showed a main effect of Phase and Time, and the interaction of Phase $\mathrm{x}$ Time 
(Table 10). The interaction indicates that the shapes of two functions were different in relative elapsed time, indicating no support for scalar timing of the sample cue durations for Group 3-27 to $3-9$.

Another conventional way to assess scalar timing of two intervals is to compare the degree of superposition of temporal functions when plotted in absolute vs. relative time. To measure the superposition of two functions in Figures 10 (the absolute time scale) and 11 (the relative time scale), an eta-squared index was computed for each subject. The index was the proportion of variance accounted for by the means of each pair of values on the two functions, and ranges from 0 to 1 , as an index of superposition of the functions (e.g., Brown, Richer, \& Doyère, 2007; Roberts, 1981). For individual subjects in both Figure 10 and 11, the index was calculated as the sum of squares for the time factor across two phases divided by the total sum of squares. Figure 12 shows group mean eta-squared values for the relative elapsed time scale and the absolute elapsed time scale separately for each group. A Group (2) x Scale (2) ANOVA of the eta-squared index showed a main effect of Group, $F(1,18)=7.79, p=0.01$, no main effect of Scale, $F(1,18)<0.01, p=0.95$, and a significant interaction of Group x Scale, $F(1,18)=6.75, p$ $=0.02$. The eta-square index for the relative scale did not differ significantly between the two groups, $t(18)=0.12, p=0.91$, but for the absolute scale, the index was greater for Group 3-27 to $3-9, t(18)=-3.54, p<0.01$. An orthogonal contrast test showed a significant difference, $F(1,18)$ $=6.75, p<0.05$, supporting the hypothesis that the degree of superposition was better on the relative scale than on the absolute scale for Group 3-9 to 3-27, but was the reverse for Group 327 to $3-9$.

In sum, the statistical analyses of normalized rate showed that for Group 3-9 to 3-27 there was a difference between the temporal patterns of response rate functions in the two phases when 
data were plotted in absolute time, but not when plotted in relative time, consistent with scalar timing of sample durations in both phases. For Group 3-27 to 3-9, there were pattern differences across phases for both absolute and relative time; however for the absolute time plot that group exhibited substantial similarity in pattern across phases. The superposition index analysis (etasquared) showed the same trend. That is, the superposition of the functions was better for relative time than for absolute time for Group 3-9 to 3-27, whereas it was better for absolute time than for relative time for Group 3-27 to 3-9.

\section{Psychometric functions in relative time}

The sample-cue response measure showed that performances in the two phases of Group 3-9 to 3-27 were different from those of Group 3-27 to 3-9. That is, the sample-cue response patterns in the 3- vs. 9-s and the 3- vs. 27-s conditions showed the scalar property in Group 3-9 to 3-27, but not in Group 3-27 to 3-9. In the following analyses, the superposition analysis is applied to a second variable, the psychometric function based on the $\mathrm{p}$ (long) measures to determine the presence of the scalar property. The time scale of the psychometric functions in Figure 5 was transformed into 7 equal-interval bins on a log scale representing relative duration for each of the two long anchor durations. Figure 13 shows the mean $\mathrm{p}$ (long) as a function of the relative duration on a log scale for Phases 1 and 2. The upper graph refers to Group 3-9 to 3-27 and the lower graph to Group 3-27 to 3-9.

The results of a mixed Group (2) x Phase (2) x Time (7) ANOVA of $\mathrm{p}($ long) can be seen in Table 11. The analysis yielded significant main effects of Group and Time, as well as Group x Time and Group x Phase interactions. In addition, there was a significant Group x Phase x Time interaction. The three-way interaction showed that the difference in the patterns of the two functions between phases depended on group. 
For Group 3-9 to 3-27, a Phase (2) x Time (7) ANOVA showed a significant main effect of Time, but no effect of phase or Time x Phase interaction (Table 12). For Group 3-27 to 3-9, the Phase (2) x Time (7) ANOVA showed a significant main effect of Phase, a main effect of Time, and a Phase $\mathrm{x}$ Time interaction (Table 13). Thus, the psychometric function plotted in relative time indicated no effect of phase (3- vs. 9-s condition and 3- vs. 27-s condition) for Group 3-9 to 3-27, consistent with the scalar property, but the functions for Group 3-27 to 3-9 differed across phases. These findings are consistent with the trends obtained in the foregoing analyses of the sample-cue responding functions.

The superposition index based on the individual subject's psychometric functions on a relative-time scale showed no difference between Group 3-9 to 3-27 and Group 3-27 to 3-9, group mean $\eta^{2}=0.96$ and $\eta^{2}=0.94$, respectively, $t(18)=1.08, p=0.29$, although the trend was similar to that observed when the superposition index was applied to the sample-cue response functions and compared on the relative time scale between two groups. For the psychometric function, the absolute time scale analysis was not available because the intermediate stimulus durations were different in the 3-vs. 9-s condition and the 3-vs. 27-s condition. 


\section{Discussion}

Experiment 2 was a systematic replication of Machado and Keen (2003; Exp. 3) with a standard operant chamber. Pigeons were exposed to the temporal bisection procedure with the 3vs 9-s and 3- vs. 27-s conditions in reverse order for two different groups. Exp. 2 yielded an effect of the long-duration anchor on the location of the BP, but the degree of the effect depended on the order of the conditions between two groups. As in Exp. 1, the BP between the 3- vs. 9-s and 3- vs. 27-s conditions differed. However, the difference in the BP between two conditions was larger for Group 3-9 to 3-27 than for Group 3-27 to 3-9, indicating that the order of the discrimination condition influenced the location of the BP. The BP was at the GM in condition 3- vs. 9-s for both groups. When the 3-vs. 9-s condition preceded the 3-vs. 27-s condition (Group 3-9 to 3-27), the BP in the 3-vs. 27-s condition was at the GM of the two anchor durations. This result was in line with the result of Exp. 1 as well as the prediction of the ratio similarity rule. On the other hand, when the 3-vs. 27-s condition was the initial condition (Group 3-27 to 3-9), the BP was less than the GM. A small influence of the long anchor duration on the location of the BP was consistent with the prediction of the Short/no short effect. The outcomes for both groups suggested that the influence of the long anchor duration on the BP depended on the order of conditions.

As in Exp. 1, the sample-cue response patterns were examined. Importantly, the shape of the response function in the initial phase was different across the two groups. The function for Group 3-9 to 3-27 showed a V-shape, whereas that for Group 3-27 to 3-9 showed a sharp decline in response rate, which remained relatively low thereafter. These response patterns in Phase 1 were similar to these in Phase 2 within a group, suggesting a primacy effect. 
Thus, in addition to Exp. 1, the present experiment showed that the location of the BP was not only a function of the short and long anchor durations but also of order of the discrimination training conditions. Depending on the order, the prediction of either the ratio similarity rule or the Short/no short hypothesis could be supported. 


\section{General Discussion}

The present study employed a temporal bisection procedure with pigeons in a standard operant chamber to investigate the effect of the duration of the long anchor cue. The dependent measures were sessions to acquisition, the location of the BP, gamma, the sample-cue response patterns, and the psychometric functions. In brief, an effect of the long duration on performance was found, but depended on the order of the conditions in the two groups. When the long anchor duration increased from $9 \mathrm{~s}$ to $27 \mathrm{~s}$, the value of the BP also increased for both groups, but the magnitude of the increase was different between the two groups. Specifically, the effect of the long anchor duration was larger for Group 3-9 to 3-27 than for Group 3-27 to 3-9, owing primarily to the BP in condition 3- vs. 27-s. When the 3- vs. 9-s condition preceded the 3- vs. 27s condition (Group 3-9 to 3-27), the BP in the 3-vs. 27-s condition was at the GM of the two anchor durations, whereas when the 3-vs. 27-s condition was presented in the initial phase (Group 3-27 to 3-9), the BP was less than the GM of those two durations.

The sample-cue response patterns showed that (1) the shape of the response function in the initial phase was similar to that in the next phase within a group, and (2) the shapes were different between groups. Moreover, the functions of Group 3-9 to 3-27 superposed better when the time axis was plotted on a relative scale than when plotted on an absolute scale, suggesting the scalar property of the sample-cue responding. For Group 3-27 to 3-9, superposition was better when the time axis was an absolute scale than when it was a relative scale. The same trend was also evident in the psychometric functions plotted on a relative time scale: For Group 3-9 to 3-27, the two functions were similar under both conditions, whereas they differed for Group 3-27 to 3-9. These findings are discussed in detail in the following sections. 


\section{Sessions to Acquisition}

The present study found that Group 3-27 to 3-9 reached the mastery criterion in fewer training sessions than did Group 3-9 to 3-27 during the initial training phase (Fig. 4). This result suggests that discrimination with a large stimulus difference, the 3-vs. 27-s condition, was acquired faster than the one with smaller stimulus difference, the 3-vs. 9-s condition in agreement with previous findings on the relation of the difference in discriminative stimuli and acquisition speed (Dinsmoor, 1995). In addition, there was a greater reduction from Phase 1 to Phase 2 in the number of sessions to acquisition for Group 3-9 to 3-27 compared to that for Group 3-27 to 3-9. The reduction in sessions to acquisition between phases suggests a transfer of training effect, thus a similar difference in sessions to acquisition between conditions was observed in phase 2, but smaller than that in phase 1.

\section{Bisection Point}

The present study found that the BP in the 3-vs 27-s condition was significantly larger than that in the 3- vs 9-s condition for both groups in Exp. 1 and Exp. 2. Thus, the present study failed to replicate Machado and Keen (2003). Using a long box and the measurement of the location of the pigeons, Machado and Keen (Exp. 3) found that the departure time, an analogue measure of the BP, did not vary when the long anchor was changed from 3- vs 9-s to 3- vs 27-s or vice versa, indicating that the change in the long anchor duration had little or no effect on the location of the departure time. The present study suggests that the findings of Machado and Keen may be specific to their use of a long box configuration of their experimental chamber or possibly to the use of the departure time as an analogue measure of the BP. The present results confirm the role of the long anchor duration in the determination of the BP. 
Platt and Davis (1983) conducted an experiment with a similar manipulation with their concurrent FI FI schedules of reinforcement procedure. Specifically, they changed the long FI value while fixing the short FI value, and measured the median switching point from the short FI schedule to the long FI schedule as well as the crossing point between the two FI response functions as an analogue measure of the BP. They found that both the median switching point and the crossing point were at the GM of the two FI values with L:S ratios of 4:1 or less. When they increased the duration of the long FI value so that the ratio exceeded 4:1, these points deviated from the GM. Their findings did not fully correspond to the results of the present study. The present study used an anchor ratio of 3:1 for the 3- vs. 9-s condition and 9:1 for the 3-vs 27$\mathrm{s}$ condition, and found that the BP was influenced by the change in the long anchor duration even when the ratio exceeded 4:1. The discrepancy between the results of their study and the present study might have been due to the difference in the sensitivity of the dependent variables in the concurrent FI FI schedule and the bisection procedure. Platt and Davis noted that the concurrent FI FI schedule was not sensitive to the detection of the midpoint when the ratio of two FI intervals became large. Another difference was that the absolute durations of the timing cue used in Platt and Davis were ten times larger than those of the present study.

The present study found that the difference in the values of the BP between the two conditions, 3-vs. 9-s and 3-vs. 27-s, depended on the order of the conditions, which differed between the groups. There was a larger difference in the BPs between the two conditions for Group 3-9 to 3-27 than for Group 3-27 to 3-9. The finding that the value of the BP was a function of order was novel and unexpected. Specifically, for the 3- vs. 27-s condition, the values of the BP varied between two groups. The BP for the 3- vs. 27-s condition was $8.7 \mathrm{~s}$, at the GM, for Group 3-9 to 3-27, whereas for Group 3-27 to 3-9, it was 6.2 s, in between the HM and the 
GM. The observation that the BP in the 3-vs. 27-s condition could vary depending on the order of the conditions has several implications. First, the influence of the long anchor duration on the location of the BP could be as strong for an anchor ratio of 9:1 as for a 3:1 ratio in the case of Group 3-9 to 3-27, which was not the case in Platt and Davis (1983) or Machado and Keen (2003). Second, the location of the BP at the GM in both the 3- vs. 9-s condition and the 3- vs. 27-s condition in Group 3-9 to 3-27 is consistent with the ratio similarity rule, whereas the small change in the BP between the two conditions in Group 3-27 to 3-9 (BP = 4.9 s in the 3- vs. 9-s condition and $6.2 \mathrm{~s}$ in the 3 - vs. 27-s condition) is similar to the performance of the pigeons in Machado and Keen (2003), where their average departure time was 4.7 s for the 3- vs. 9-s condition and $5.6 \mathrm{~s}$ for the 3- vs. 27-s condition. The performance of Group 3-27 to 3-9 conforms closely to the prediction of the Short/no short hypothesis. And third, neither the ratio similarity rule nor the Short/no short hypothesis alone can accommodate the order effect since both hypotheses predict a single BP value for a given pair of anchor durations without bias. The PLM accounted for moderate proportion of the variance in BP $\left(r^{2}=.53\right)$, consistent with Machado and Keen (2003) who observed a close match between their BPs and the PLM predictions. Although PLM can account for differences in BP based on corresponding values of gamma, it is not clear how the model accounts for differences in gamma among conditions.

To summarize, the initial discrimination condition produced differential performance that was consistent with either the ratio similarity rule or the Short/no short hypothesis. In the following sections, other measures including the sample-cue response patterns and the psychometric functions are examined to consider the possibility that the exposure to different initial conditions may have engaged different mechanisms underlying performances in the temporal bisection procedure. 


\section{Sample-cue responding}

The pecking responses to the timing cue, prior to the presentation of the comparison keys, were reported. There were three interesting findings obtained with this measure. The first was that the sample-cue response rate changed as a function of elapsed time. Although the patterns of sample-cue responding were different between the two groups, response rates changed significantly with elapsed time for both groups, indicating that the sample-cue responding was sensitive to elapsed time. Second, the sample-cue responding patterns were similar between the two phases within each group. For Group 3-9 to 3-27, the shapes of the response rate functions in the 3-vs. 9-s condition and the 3-vs. 27-s condition were both V-shaped, showing an initial decrease followed by an increase in response rate at the end of the elapsed sample-cue period. For Group 3-27 to 3-9, the shapes of the sample-cue response rate functions in each condition showed a decrease in response rate to a lower level that was maintained until the end of the sample-cue presentation. Depending on the condition in the initial phase, the sample-cue response patterns differed, and those patterns transferred to the next phase for each group. Third, for Group 3-9 to 3-27, the rate-normalized response patterns in the 3- vs. 9-s condition and the 3vs. 27-s condition showed better superposition when the time axis was normalized than when the time axis was not normalized, providing evidence of scalar timing of the anchor durations. Together with the finding that the BP was at the GM in both the 3- vs. 9-s and 3- vs. 27-s conditions, the sample-cue responding patterns indicated that both the short and long anchor durations acquired control of the subjects' behavior during the task, consistent with the ratio similarity rule. In contrast, for Group 3-27 to 3-9, superposition was better when the time axis was absolute than when it was normalized. With the finding that the difference in the BPs between two conditions for this group was smaller than that of the other group, the sample-cue 
responding patterns in this group suggested little control of behavior by the long anchor duration, and that the short anchor duration exerted a dominant influence on behavior, consistent with the short/no short rule.

Performance during the sample cue is diagnostic of temporal control mechanism in the present study. Little attention has been paid to that behavior in the temporal bisection literature. A few exceptions are Zentall and his colleagues (Zentall, Friedrich, \& Clement, 2006; Zentall \& Singer, 2008) who were interested in the effect of different response requirements on the timing cue on the location of the BP. They observed that pigeons pecked at the timing cue even without response requirements, and compared the response rates between short- and long-anchor trials. They did not find a difference in rate between the two trial types, and did not analyze responding in relation to elapsed time

In other paradigms, behavior similar to sample-cue responding was reported. Richelle and Lejeune (1980) reviewed behavior patterns aside from the operant observed during the presentation of schedules including FI or differential reinforcement of low rate. These behaviors are called 'adjunctive behavior,' 'collateral behavior' or 'interim behavior' in contrast to the operant or 'terminal response' that occurs in close contiguity with the reinforcer (Staddon \& Simmelhag, 1971). Such collateral behavior often showed a systematic pattern as a function of the temporal aspect of the experimental manipulations, encouraging some researchers to infer that collateral behavior 'mediates' the operant. For example, Ferster and Skinner (1957) hypothesized that an organism develops chained responding, which functions as the source of stimulus control to occasion or mediate the operant in a given cycle of a FI schedule. Some theories of timing behavior such as BeT (Killeen \& Fetterman, 1988) and LeT (Machado, 1997) hypothesized that the sequence of responses during an inter-reinforcement interval mediates the 
operant behavior. However, it is difficult to identify collateral behavior as the cause of, or a part of the causal chain of, the operant since (1) it is not a variable of which an experimenter has a direct control and (2) it is difficult if not impossible to observe all possible types of collateral behavior, including covert/private behavior during an experiment, which renders the mediating hypothesis unfalsifiable. Furthermore, Dews (1962) manipulated variables that disrupted or eliminated collateral behavior in a FI schedule and found that collateral behavior did not seem to mediate the temporal pattern of the operant.

Sample-cue responding in the present study could be seen as a variant of collateral behavior. However, sample-cue responding is not viewed as 'mediating' the operant in the temporal bisection procedure. Rather, it is more plausibly viewed as one manifestation of a common mechanism responsible for performance in both the sample and comparison phases of a temporal bisection trial.

Specifically, it is proposed that there were different sources of stimulus control established for each group in the initial discrimination problem. For Group 3-9 to 3-27, both the short and long anchor stimuli influenced sample-cue responding and $\mathrm{p}$ (long) in the comparison period, whereas for Group 3-27 to 3-9, control was exerted by the short anchor duration alone. That is, the initial exposure to two different discrimination problems produced different sources of stimulus control in both the sample and comparison components of the bisection trial.

The notion that different aspects of the environment might acquire stimulus control in discrimination training is not new. For example, Honig and Urcuioli (1981) reviewed studies of the stimulus generalization procedure. In their review, they discussed the effects of procedures called interdimensional discrimination training and extradimensional discrimination training on the shape of the stimulus generalization gradient. In such discrimination training, the 
experimenter presents a compound stimulus in training, and measures the shape of the separate gradients in testing to investigate which aspects of the compound stimulus acquired control over the organisms' behavior. The research showed that, during discrimination training, more than one aspect of the environment, including the subjects' own behavior pattern, could acquire control of behavior. The findings in the present study encourage the view that depending on the initial discrimination training, either both the short and long anchor stimuli or the short anchor alone could acquire control over performance in the temporal bisection procedure. This idea is further elaborated below.

\section{Psychometric function}

The psychometric function based on the $\mathrm{p}$ (long) measure also indicated different patterns of behavior between two groups. When plotted on a relative time scale, the psychometric functions for the two conditions superposed better for Group 3-9 to 3-27 compared to those of Group 3-27 to 3-9. This difference indicates that $\mathrm{p}($ long) behavior in Group 3-9 to 3-27 manifested the scalar property, but that behavior for Group 3-27 to 3-9 did not.

\section{Implications}

This study was suggested by a number of observations in the temporal bisection literature that the BP was not always found at the GM of the two anchor durations, contrary to the original observations of Church and Deluty (1971). The present study found that one factor that influenced the location of the BP was the initial discrimination condition, and it is suggested that differential performance under different initial discrimination conditions could be understood in terms of the ratio similarity rule or the Short/no short hypothesis. That is, depending on the initial discrimination training, the degree of stimulus control by the short and long anchors differed. In addition, once stimulus control was established in the initial discrimination phase, that source of 
control was transferred to the following discrimination phase with different set of anchors. When the initial condition was 3- vs. 9-s, both the short and long anchor durations acquired control over keypeck performance in the sample (sample-cue responding) and comparison (p[long]) components of a trial, whereas when the initial condition was 3- vs. 27-s, the short anchor stimulus acquires a primary stimulus control and the long anchor's influence was minor. This difference in the degree of stimulus control established by the long duration in the initial discrimination phase might have been due to the difference in the ratio between two anchor pairs, 3:1 vs. 9:1, although this variable was confounded with the difference in the absolute duration of the long anchors. With a small ratio such as 3:1, both the short and long anchors acquire control over behavior in a manner suggested by the ratio similarity rule, and the scalar property for both sample-cue responding and the psychometric function. On the other hand, with a large ratio such as 9:1, the short anchor dominates in the control of behavior, with weak if any control by the long anchor, in line with the Short/no short hypothesis. Subsequently, the established source of stimulus control transfers to the following discrimination training regardless of the ratio of the anchors.

This idea concerning the source of stimulus control is similar to Platt and Davis' (1983) interpretation of their data. They reported that with a small anchor ratio (less than 4:1 ratio), the BP typically fell at around the GM of two FI intervals in their concurrent FI FI schedules paradigm. Their results were consistent with those of Church and Deluty (1971) and the prediction of the ratio similarity rule. With a larger ratio, they observed that both the crossing point of the two FI functions and the median switching point departed from the GM of the two intervals (Exp. 2.). They indicated that when two FI values were larger than a ratio of 4:1, the two FI schedules exerted independent control of behavior and did not compete for responding. 
Their conclusion was that the ratio similarity rule can account for performance under the ratio of 4:1, and beyond that ratio, there would be no comparison of two durations (a similar argument can be seen in Church, 1993). Thus, Platt and Davis proposed a two-fold hypothesis of performance under the bisection procedure: the ratio similarity rule can account for performance under 4:1 ratio and the independent control of behavior takes over behavior beyond that ratio.

Two aspects of the data from the present study did not conform to Platt and Davis' hypothesis. One was that the present study showed that, after the initial 3-vs. 9-s condition, the $\mathrm{BP}$ in the 3- vs. 27-s condition was found to be at the GM of two anchor durations, indicating that stimulus control by the long anchor duration was evident even when the ratio was as large as 9:1, and suggesting that the ratio similarity rule can account for the performance even beyond a ratio of 4:1. The second was that, although the BP in the 3- vs. 9-s condition for both groups was at the GM of the two anchors, sample-cue patterns between two groups were different, suggesting that the nature of stimulus control in this condition might be different between two groups, and that the 4:1 limit on the control by both short and long anchors may not always apply.

Machado and Keen (2003) indicated that the short anchor stimulus always acquires strong and independent control over the BP and departure time regardless of the ratio of the anchor pairs in the temporal bisection task - a statement of the Short/no short hypothesis. This idea was based on the observation that the BP and the departure time were at (Exp. 2) or less than (Exp. 1) the GM of two anchor durations. When the long duration increased from $9 \mathrm{~s}$ to 27 s, or from $27 \mathrm{~s}$ to $81 \mathrm{~s}$ in Exp. 3, the departure time did not increase accordingly. As the departure time shifted only when the short duration increased, they hypothesized that the BP and the departure time depended only on the short duration. This hypothesis can predict the results of 
both Church and Deluty (1977) and Platt and Davis (1983) without introducing multiple assumptions (two different processes applying below and beyond an anchor ratio of 4:1), although it does not provide a reason that the BP in conditions where the ratio of two anchors are 4:1 or less should approximate the GM of two anchors. In relation to the present study, the Short/no short hypothesis cannot account for the order effect, where there was a larger difference in the BP for Group 3-9 to 3-27 than for Group 3-27 to 3-9 when the long anchor changed. Machado and Keen (2003) did not find an order effect when they counterbalanced the order of discrimination training between 3- vs. 9-s and 3- vs. 27-s conditions or between 9- vs. 27-s and 9- vs. 81-s conditions in Exp. 3. The lack of the order effect may be attributed to differences in the experimental procedure compared to the present study. One difference was Machado and Keen's use of the long chamber, which might have interfered with the development of the control by the long anchor, possibly by the induction of gross locomotor behavior patterns during the sample cue. They reported that pigeons moved to the 'short' side of the box at the onset of the timing cue and moved to the 'long' side of the box as the short duration elapsed. Thus, it might be the case that the control by the long anchor duration was overshadowed by the subject's stereotyped behavior pattern at the time of the departure from the short cue regardless of what the initial discrimination condition was. Since departure time was taken as an analogue measure of the BP, the effect of the long anchor might have not been detected. In the standard operant chamber where the timing cue and the comparison side keys were closely placed on the front panel, the development of the stereotyped behavior pattern might have not been as robust as in the long chamber or competed with the control by the long anchor cue, thus permitting the BP measure to be sensitive to the differences in the order of conditions. 
It is also possible that Machado and Keen did not find the order effect due to the lack of power owing to the number of subjects, $N=5$ ( 3 birds for one order and 2 birds for the counterorder) in Exp. 3. This possibility, however, has little support. They reported that there was a slight difference in the departure time for the pigeons $(n=2)$, who were exposed to the order 3 vs. 27-s followed by 3- vs. 9-s, whereas there was no difference for the other order from 3- vs. 9s to 3- vs. 27-s. The direction of the small difference between two orders was the reverse of the present study's results.

Other researchers who used anchor durations larger than 4:1 with non-human animals are Raslear (1983; 1985) and Siegel (1986). Raslear (1983) found the BP at 1.4 s, not-significantly different from the GM of two anchor durations, 0.1- vs. 10-s, with the ratio of 10:1. This result is not consistent with the prediction advanced here that the short/no-short rule should apply with anchor ratios greater than 4:1, yielding a BP that is less than the GM. Raslear used the 0.1- vs. 10-s condition as the initial condition, but he did not find the BP to be less than the GM. At the same time, as the short anchor duration was $0.1 \mathrm{~s}$ and the BP was at $1.4 \mathrm{~s}$, it is open to question whether a BP smaller than $1 \mathrm{~s}$ is readily measurable. In contrast, Siegel (1986) used anchor values of 1- vs. 16-s and found that the BP was less than the GM of the two durations, although he also presented the test stimuli that were shorter than or longer than the anchor values. Siegel attributed the deviation of the BP from the GM to the presence of the test stimuli outside the anchor value range, but it could well be due to the use of a large anchor ratio, which might have produced performance consistent with the Short/no short hypothesis.

\section{Conclusion}

The present study investigated the effect of the long anchor duration on the location of the $\mathrm{BP}$ in the temporal bisection procedure with pigeons in a standard operant chamber as a 
systematic replication of Machado and Keen (2003). Unlike Machado and Keen, when the long anchor duration value changed between the 3- vs. 9-s and the 3-vs. 27-s conditions, the BP also changed. More importantly, the degree of the change in the BP between the two conditions differed across two groups exposed to different sequences of conditions. It is suggested that the anchor values of the initial condition influenced the location of the BP for both phases. When the initial phase was the 3-vs. 9-s condition, the BPs for both conditions were at the GM of the two anchor durations, consistent with the ratio similarity rule. When the initial phase was the 3 - vs. 27-s condition, the BP was smaller than the GM for the 3-vs. 27-s condition and at the GM for the 3- vs. 9-s condition, consistent with the short/no-short hypothesis. Neither the ratio similarity rule nor the short/no short hypothesis alone predicted these effects.

Evidence from sample-cue response patterns and the psychometric function suggested that the two groups might have performed this task differently. Sample-cue responding measures showed different temporal patterns of responding between two groups. For Group 3-9 to 3-27, the response rate functions during both the 3-vs. 9-s condition and the 3-vs. 27-s condition revealed scalar timing of the anchor durations, overlapping better when the time axis was normalized compared to when it was not. The evidence of the scalar property suggested that this group's behavior was controlled by both the short and long anchor durations. On the other hand, for Group 3-27 to 3-9, the sample-cue response rate functions superposed better with an absolute-time axis than with the relative-time axis, indicating little or no control by the long anchor duration. Psychometric functions on the relative-time axis also showed a better superposition for Group 3-9 to 3-27 than for Group 3-27 to 3-9, supporting the view that the initial condition differentially influenced performance of two groups. The results of the present study suggest that the effect of the long anchor duration on the location of the BP depends on the 
order of conditions in the temporal bisection task with the standard operant chamber, suggesting a primacy effect. Once established, temporal control by both short and long anchors, or by the short anchor alone, transferred to a second problem with a different long anchor. The present findings establish history of reinforcement as a variable worthy of further investigation in the temporal bisection task. 
Table 1.

The scheme of Experiment 1 for two groups (rows) and four phases.*

\begin{tabular}{ccccc}
\hline & Phase 1 & Phase 2 & Phase 3 & Phase 4 \\
\hline Group Exp & $3-9$ & $3-27$ & $3-13.5$ & $3-27$ \\
Group Control & $3-9$ & $3-27$ & $3-27$ & $3-27$ \\
\hline
\end{tabular}

* The numbers in each cell show the anchor stimulus durations. The left and right numbers are the short and long anchor durations, respectively. All units are seconds. 
Table 2 .

Group x Phase mixed analysis of variance of BP in the four phases of Exp. 1.

\begin{tabular}{lccccc}
\hline Source & $d f$ & $S S$ & $M S$ & $F$ & $p$ \\
\hline Group & 1 & 2.58 & 2.58 & 0.31 & 0.59 \\
Error & 10 & 83.53 & 8.35 & & \\
Phase & 3 & 47.34 & 15.78 & 6.89 & $<.01$ \\
Group x Phase & 3 & 12.39 & 4.13 & 1.8 & 0.17 \\
Error & 30 & 68.83 & 2.29 & & \\
\hline
\end{tabular}


Table 3.

The scheme of Experiment 2 for two groups (rows) and two phases*

\begin{tabular}{lcc}
\hline & Phase 1 & Phase 2 \\
\hline Group 3-9 to 3-27 & $3-9$ & $3-27$ \\
Group 3-27 to 3-9 & $3-27$ & $3-9$ \\
\hline
\end{tabular}

* The numbers in each cell show the anchor stimulus durations. The left and right numbers are the short and long anchor durations, respectively. All units are seconds. 


\section{Table 4.}

The bisection point (BP), the geometric mean (GM), and the harmonic mean (HM) in each phase for each group of Experiment 2.

\begin{tabular}{ccc}
\hline & Phase 1 & Phase 2 \\
\hline Group 3-9 to 3-27 & $3-9$ & $3-27$ \\
\hline Obtained BP & 5.22 & 8.73 \\
GM & 5.20 & 9.00 \\
HM & $4.50^{*}$ & $5.40^{*}$ \\
\hline Group 3-27 to 3-9 & $3-27$ & $3-9$ \\
\hline Obtained BP & 6.24 & 4.96 \\
GM & $9.00^{*}$ & 5.20 \\
HM & $5.40^{*}$ & $4.50^{*}$ \\
\hline
\end{tabular}

Note. p-values $<.05$ are marked by * after the value of the GM or the HM if these values are statistically different from the obtained BP of that condition. All numbers are in seconds. 
Table 5 .

Group $x$ Phase $x$ Time analysis of variance of normalized rate for the sample-cue response for an absolute time scale in Exp. 2

\begin{tabular}{lccccc}
\hline Source & $d f$ & $S S$ & $M S$ & $F$ & $p$ \\
\hline Group & 1 & 0.078 & 0.078 & 0.64 & 0.44 \\
Error & 18 & 2.213 & 0.122 & & $<.01$ \\
Time & 8 & 12.374 & 1.547 & 15.02 & $<.01$ \\
Group x Time & 8 & 6.196 & 0.775 & 7.52 & 0.55 \\
Error & 144 & 14.828 & 0.103 & & 0.41 \\
Phase & 1 & 0.026 & 0.026 & 0.37 & $<.01$ \\
Group x Phase & 1 & 0.05 & 0.05 & 0.71 & \\
Error & 18 & 1.263 & 0.070 & & \\
Phase x Time & 8 & 2.403 & 0.3 & 7.18 & \\
Group x Phase x Time & 8 & 2.923 & 0.365 & 8.73 & \\
Error & 144 & 6.027 & 0.041 & & \\
\hline
\end{tabular}


Table 6.

Phase $x$ Time analysis of variance of normalized rate for the sample-cue response for an absolute time scale for Group 3-9 to 3-27 in Exp. 2

\begin{tabular}{lccccc}
\hline Source & $d f$ & $S S$ & $M S$ & $F$ & $p$ \\
\hline Phase & 1 & 0.002 & 0.002 & 0.03 & 0.88 \\
Error & 9 & 0.662 & 0.074 & & \\
Time & 8 & 1.402 & 0.175 & 1.39 & 0.21 \\
Error & 72 & 9.045 & 0.126 & & \\
Phase x Time & 8 & 5.013 & 0.627 & 9.51 & $<.01$ \\
Error & 72 & 4.743 & 0.066 & & \\
\end{tabular}


Table 7 .

Phase $x$ Time analysis of variance of normalized rate for the sample-cue response for an absolute time scale for Group 3-27 to 3-9 in Exp. 2

\begin{tabular}{lccccc}
\hline Source & $d f$ & $S S$ & $M S$ & $F$ & $p$ \\
\hline Phase & 1 & 0.074 & 0.074 & 1.11 & 0.32 \\
Error & 9 & 0.602 & 0.067 & & \\
Time & 8 & 17.169 & 2.146 & 26.72 & $<.01$ \\
Error & 72 & 5.782 & 0.08 & & \\
Phase x Time & 8 & 0.312 & 0.039 & 2.19 & 0.04 \\
Error & 72 & 1.284 & 0.018 & & \\
\hline
\end{tabular}


Table 8 .

Group $x$ Phase $x$ Time analysis of variance of normalized rate for the sample-cue response on a relative time scale in Exp. 2.

\begin{tabular}{lccccc}
\hline Source & $d f$ & $S S$ & $M S$ & $F$ & $p$ \\
\hline Group & 1 & 1.345 & 1.344 & 7.24 & 0.02 \\
Error & 18 & 3.341 & 0.186 & & $<.01$ \\
Time & 8 & 4.765 & 0.596 & 5.52 & $<.01$ \\
Group x Time & 8 & 10.343 & 1.293 & 11.97 & \\
Error & 144 & 15.549 & 0.108 & & 0.04 \\
Phase & 1 & 0.516 & 0.516 & 4.93 & 0.61 \\
Group x Phase & 1 & 0.028 & 0.028 & 0.27 & $<.01$ \\
Error & 18 & 1.883 & 0.105 & & \\
Phase x Time & 8 & 1.194 & 0.149 & 3.51 & \\
Group x Phase x Time & 8 & 3.004 & 0.376 & 8.84 & \\
Error & 144 & 6.114 & 0.042 & & \\
& & & & & \\
& & & & & \\
\end{tabular}


Table 9.

Phase $x$ Time analysis of variance of normalized rate for the sample-cue response for a relative time scale for Group 3-9 to 3-27 in Exp. 2

\begin{tabular}{lccccc}
\hline Source & $d f$ & $S S$ & $M S$ & $F$ & $p$ \\
\hline Phase & 1 & 0.151 & 0.151 & 1.11 & 0.32 \\
Error & 9 & 1.226 & 0.136 & & \\
Time & 8 & 4.184 & 0.523 & 3.53 & $<.01$ \\
Error & 72 & 10.658 & 0.148 & & 0.26 \\
Phase x Time & 8 & 0.574 & 0.072 & 1.29 & \\
Error & 72 & 4.015 & 0.056 & & \\
\hline
\end{tabular}


Table 10.

Phase $x$ Time analysis of variance of normalized rate for the sample-cue response for a relative time scale for Group 3-27 to 3-9 in Exp. 2

\begin{tabular}{lccccc}
\hline Source & $d f$ & $S S$ & $M S$ & $F$ & $p$ \\
\hline Phase & 1 & 0.393 & 0.393 & 5.38 & 0.05 \\
Error & 9 & 0.657 & 0.073 & & \\
Time & 8 & 10.924 & 1.366 & 20.10 & $<.01$ \\
Error & 72 & 4.891 & 0.068 & & $<.01$ \\
Phase x Time & 8 & 3.624 & 0.453 & 15.54 & \\
Error & 72 & 2.099 & 0.029 & & \\
\hline
\end{tabular}


Table 11.

Group $x$ Phase $x$ Time analysis of variance of $p$ (long) for the psychometric function on a relative time scale in Exp. 2

\begin{tabular}{|c|c|c|c|c|c|}
\hline Source & $d f$ & $S S$ & $M S$ & $F$ & $p$ \\
\hline Group & 1 & 0.444 & 0.444 & 12.64 & $<.01$ \\
\hline Error & 18 & 0.632 & 0.035 & & \\
\hline Time & 6 & 35.33 & 5.89 & 752.42 & $<.01$ \\
\hline Group x Time & 6 & 0.349 & 0.058 & 7.43 & $<.01$ \\
\hline Error & 108 & 0.845 & 0.007 & & \\
\hline Phase & 1 & 0.081 & 0.081 & 2.78 & 0.11 \\
\hline Group x Phase & 1 & 0.210 & 0.210 & 7.24 & 0.01 \\
\hline Error & 18 & 0.522 & 0.029 & & \\
\hline Phase $\mathrm{x}$ Time & 6 & 0.123 & 0.021 & 1.71 & 0.13 \\
\hline Group x Phase x Time & 6 & 0.374 & 0.062 & 5.18 & $<.01$ \\
\hline Error & 108 & 1.299 & 0.012 & & \\
\hline
\end{tabular}


Table 12.

Phase $x$ Time analysis of variance of $p$ (long) for the psychometric function on a relative time scale for Group 3-9 to 3-27 in Exp. 2

\begin{tabular}{lccccc}
\hline Source & $d f$ & $S S$ & $M S$ & $F$ & $p$ \\
\hline Phase & 1 & 0.015 & 0.015 & 0.48 & 0.51 \\
Error & 9 & 0.283 & 0.031 & & \\
Time & 6 & 17.664 & 2.944 & 394.39 & .01 \\
Error & 54 & 0.403 & 0.007 & & 0.63 \\
Phase x Time & 6 & 0.057 & 0.009 & 0.73 & \\
Error & 54 & 0.706 & 0.013 & & \\
\hline
\end{tabular}


Table 13.

Phase $x$ Time analysis of variance of $p$ (long) for the psychometric function on a relative time scale for Group 3-27 to 3-9 in Exp. 2

\begin{tabular}{lccccc}
\hline Source & $d f$ & $S S$ & $M S$ & $F$ & $p$ \\
\hline Phase & 1 & 0.276 & 0.276 & 10.4 & 0.01 \\
Error & 9 & 0.239 & 0.026 & & \\
Time & 6 & 18.015 & 3.002 & 366.73 & $<.01$ \\
Error & 54 & 0.442 & 0.008 & & $<.01$ \\
Phase x Time & 6 & 0.44 & 0.073 & 6.67 & \\
Error & 54 & 0.593 & 0.011 & & \\
\hline
\end{tabular}




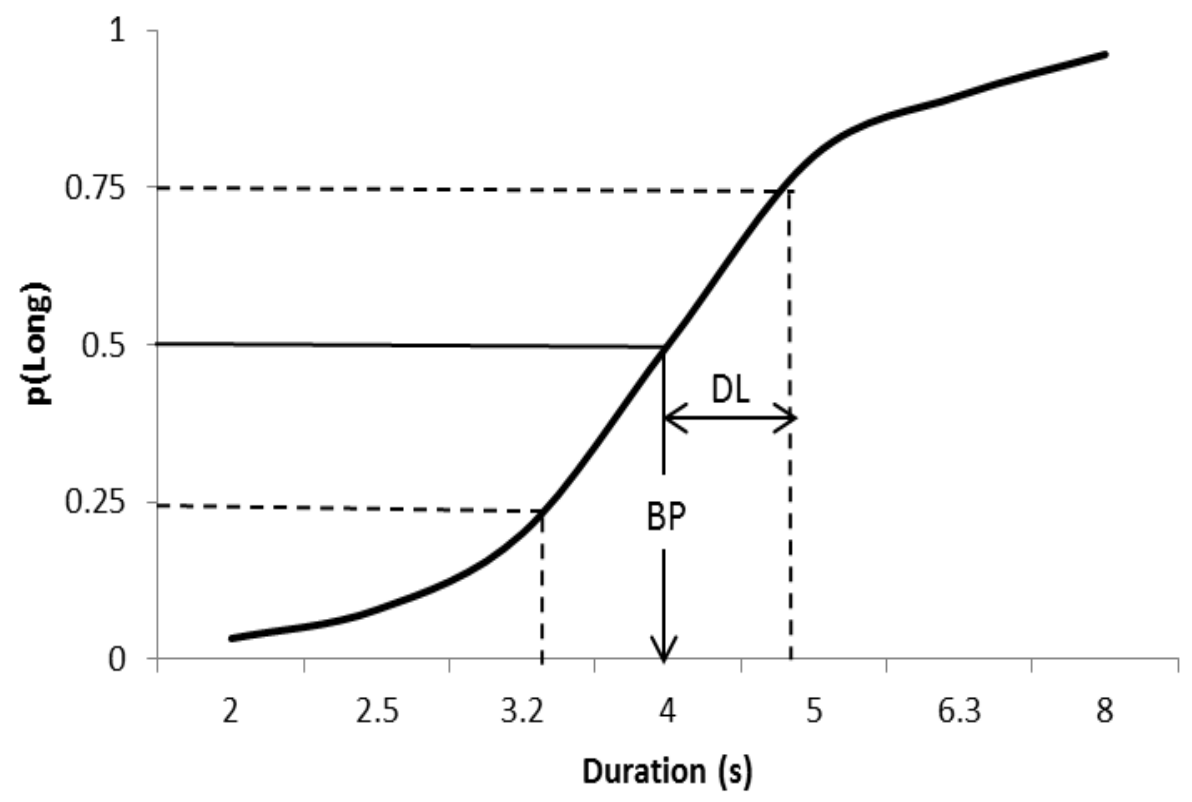

Figure 1. Hypothetical performance of a participant plotted as the probability of a 'long' response as a function of duration on a log scale in the temporal bisection procedure. 


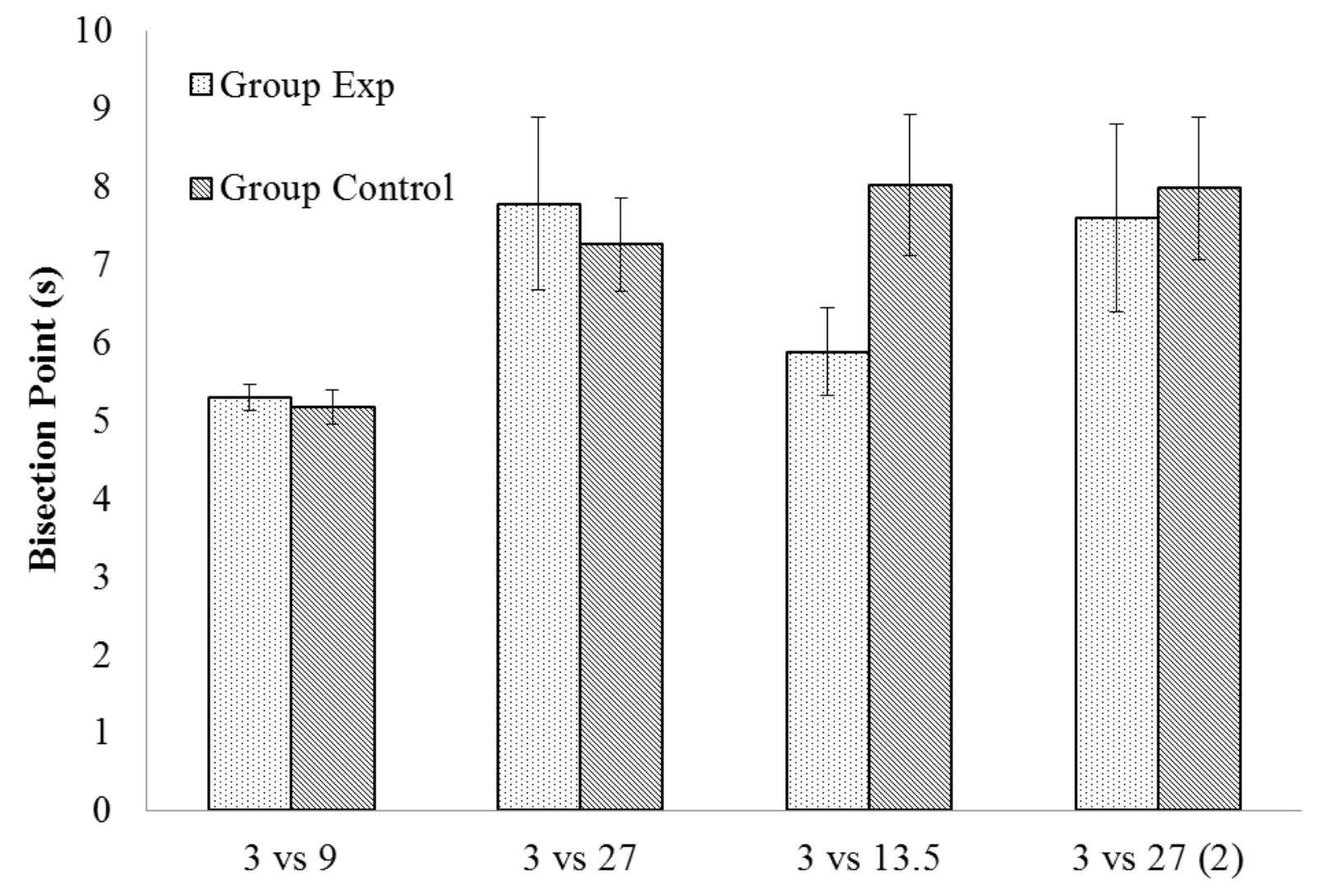

Figure 2. The group mean bisection point $( \pm S E M)$ as a function of phases for both groups in Exp. 1. The abscissa indicates values of short and long anchors (s) in each phase for Group Exp. The values of short and long anchors were 3 vs 9 in phase 1 and 3 vs 27 in all subsequent phases for Group Control. 


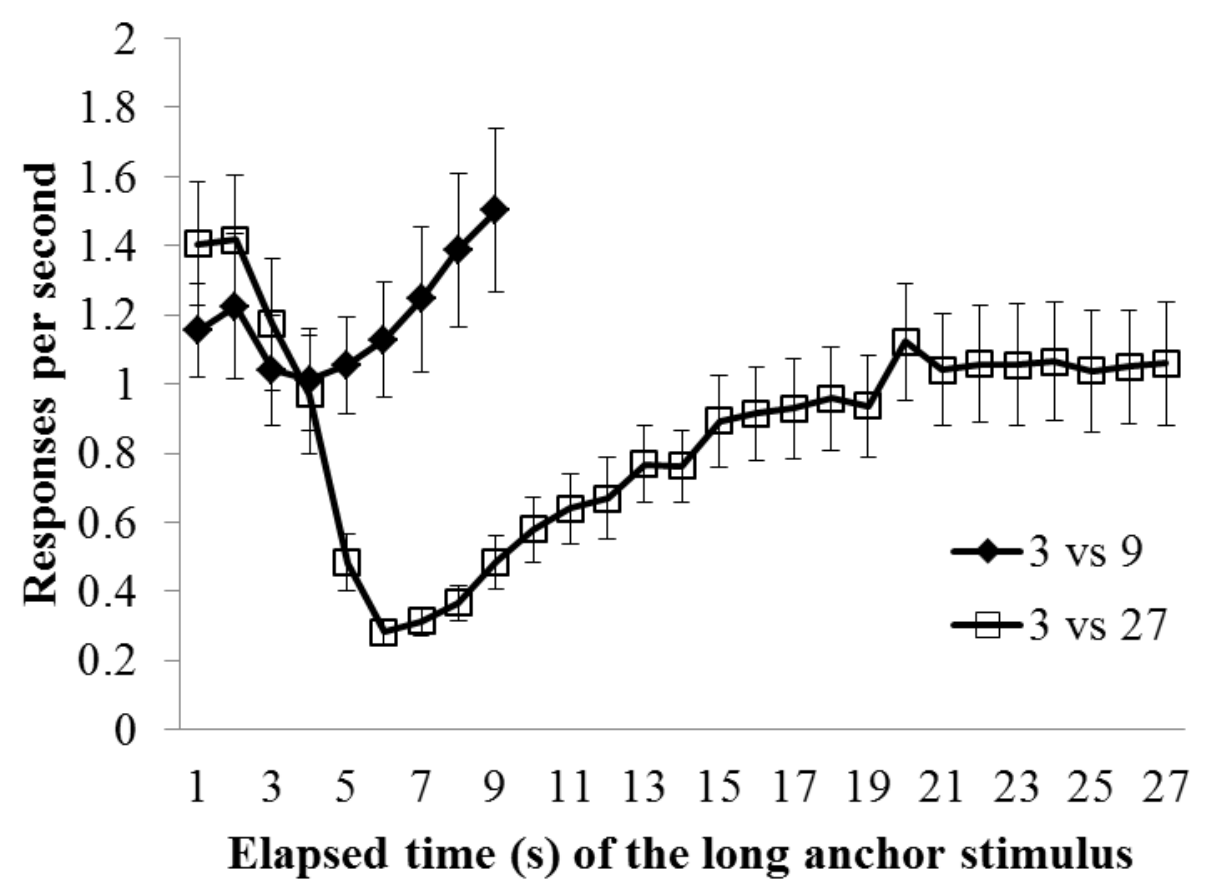

Figure 3. Group mean responses per second $( \pm S E M)$ as a function of elapsed time of the long anchor stimulus for the average across three groups for the first two phases in Exp. 1. 


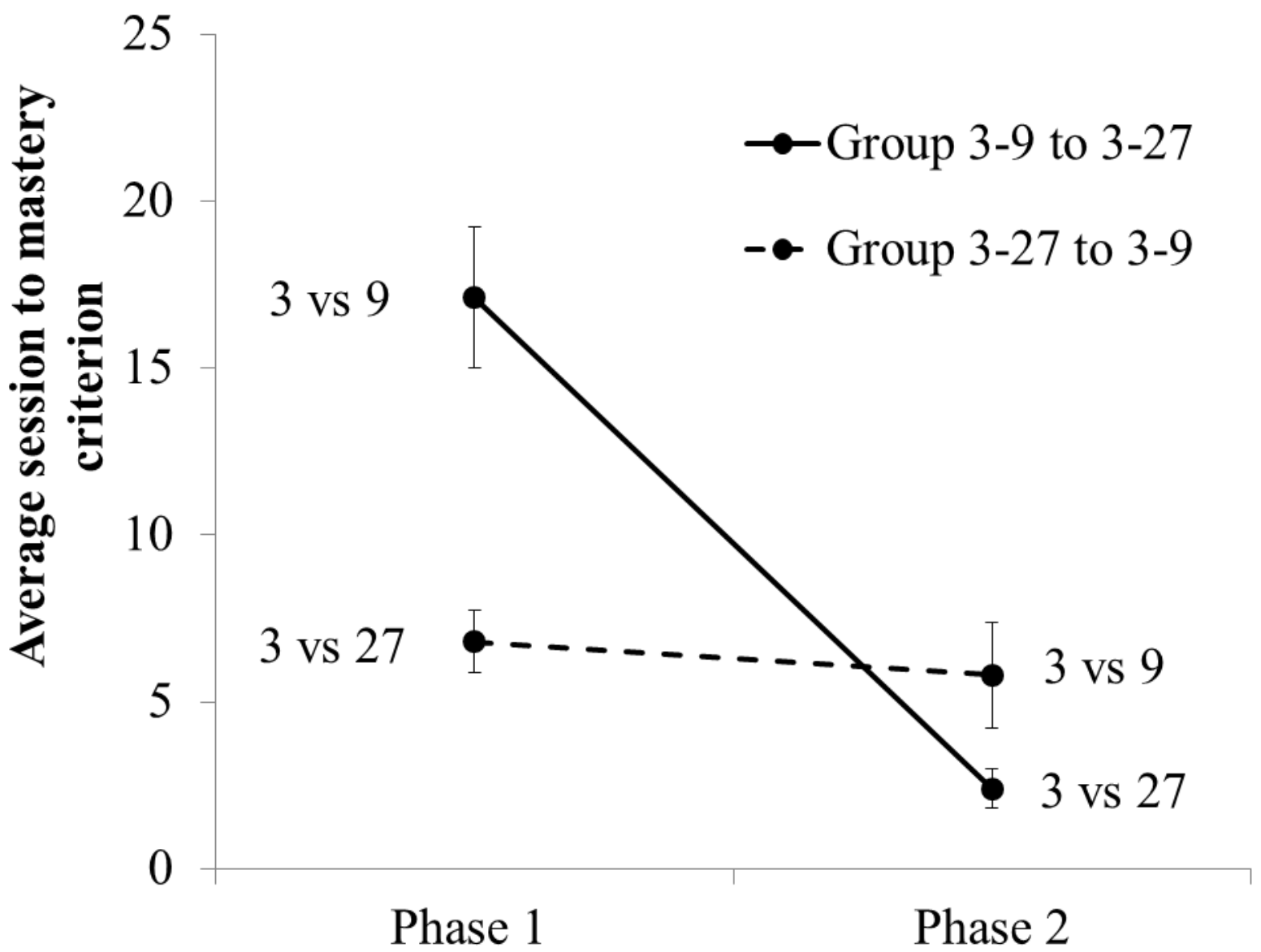

Figure 4. Group mean number of sessions to mastery criterion $( \pm S E M)$ as a function of phases for Group 3-9 to 3-27 and Group 3-27 to 3-9 in Exp. 2. Durations of short and long anchors are shown adjacent to data points. 

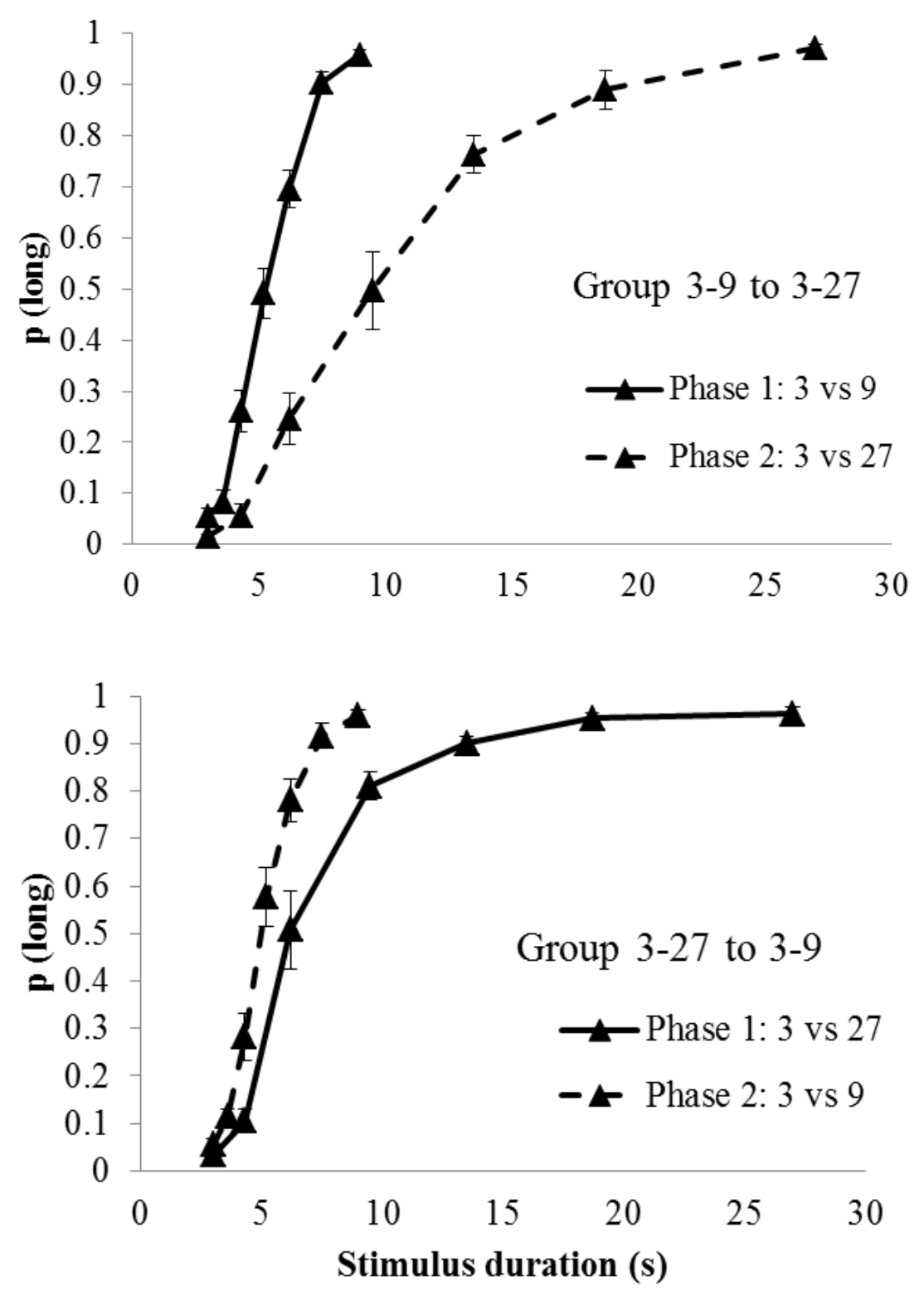

Figure 5. Group mean probability of a "Long" response $( \pm S E M)$ in each phase as a function of stimulus duration in Exp. 2. The top graph refers to Group 3-9 to 3-27 and the bottom graph to Group 3-27 to 3-9. 


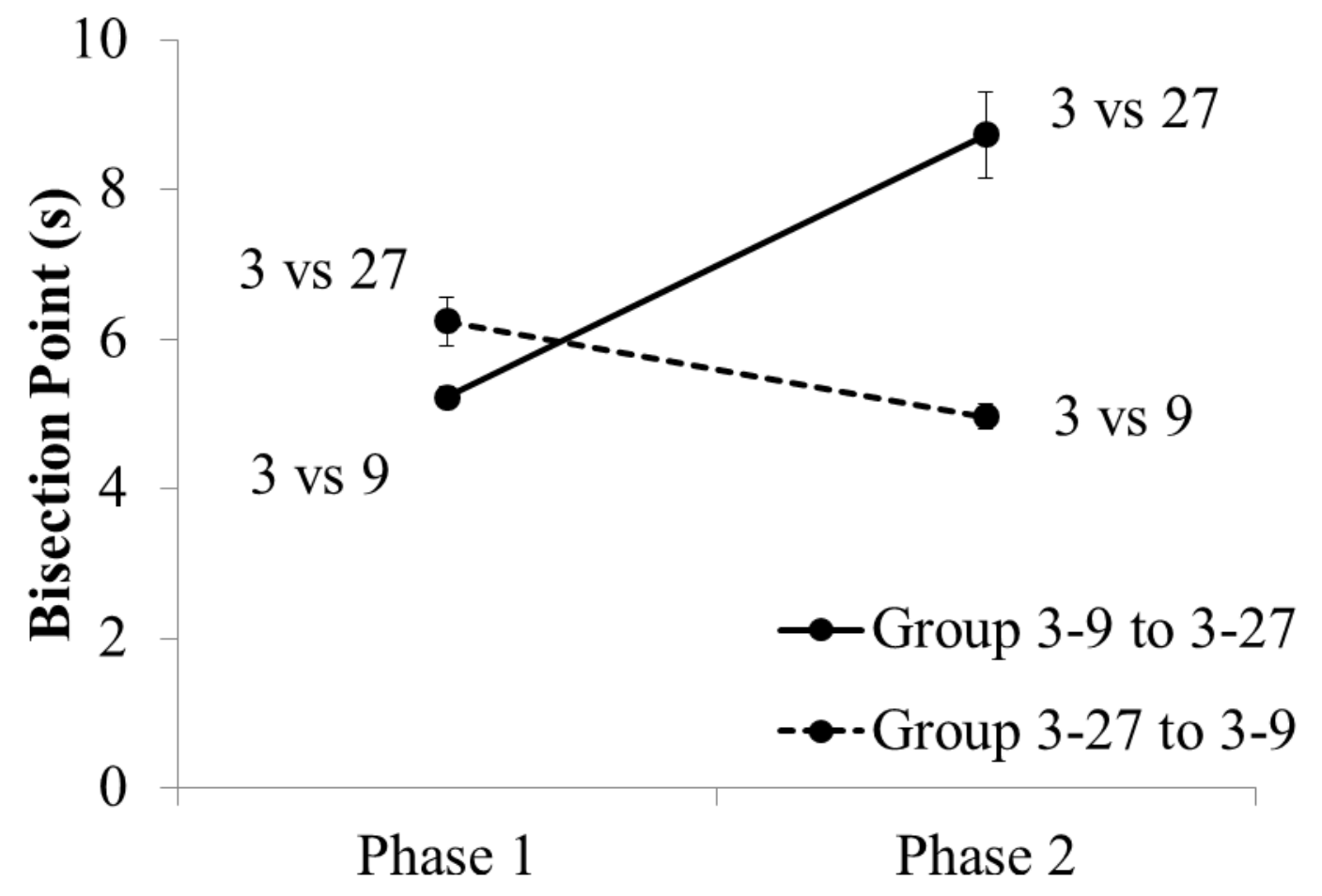

Figure 6. Group mean bisection point $( \pm S E M)$ as a function of phase for Group 3-9 to 3-27 and for Group 3-27 to 3-9 in Exp. 2. Durations of short and long anchors are shown adjacent to data points. 


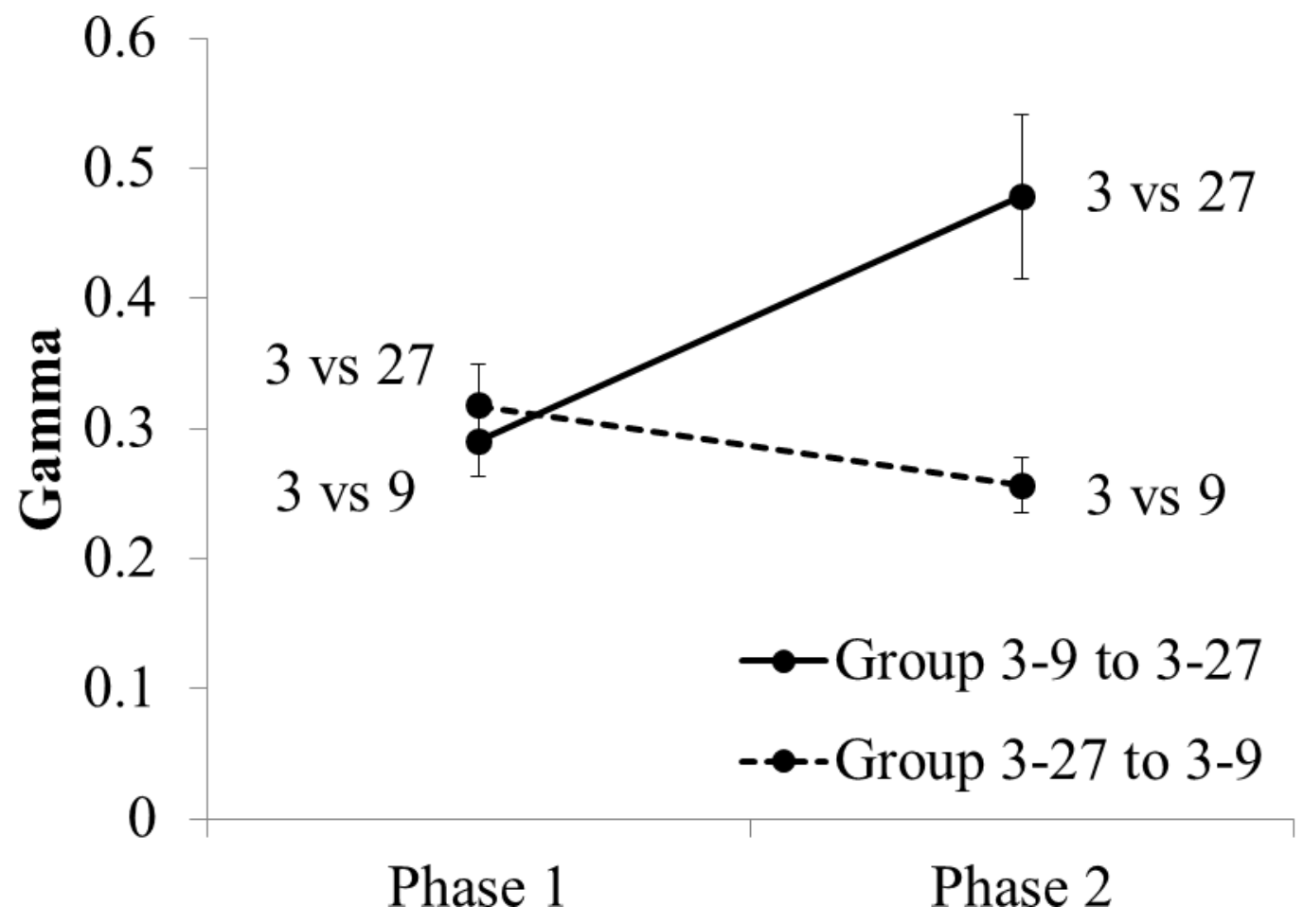

Figure 7. Group mean gamma $( \pm S E M)$ as a function of phase for Group 3-9 to 3-27 and Group 3-27 to 3-9 in Exp. 2. Durations of short and long anchors are shown adjacent to data points. 


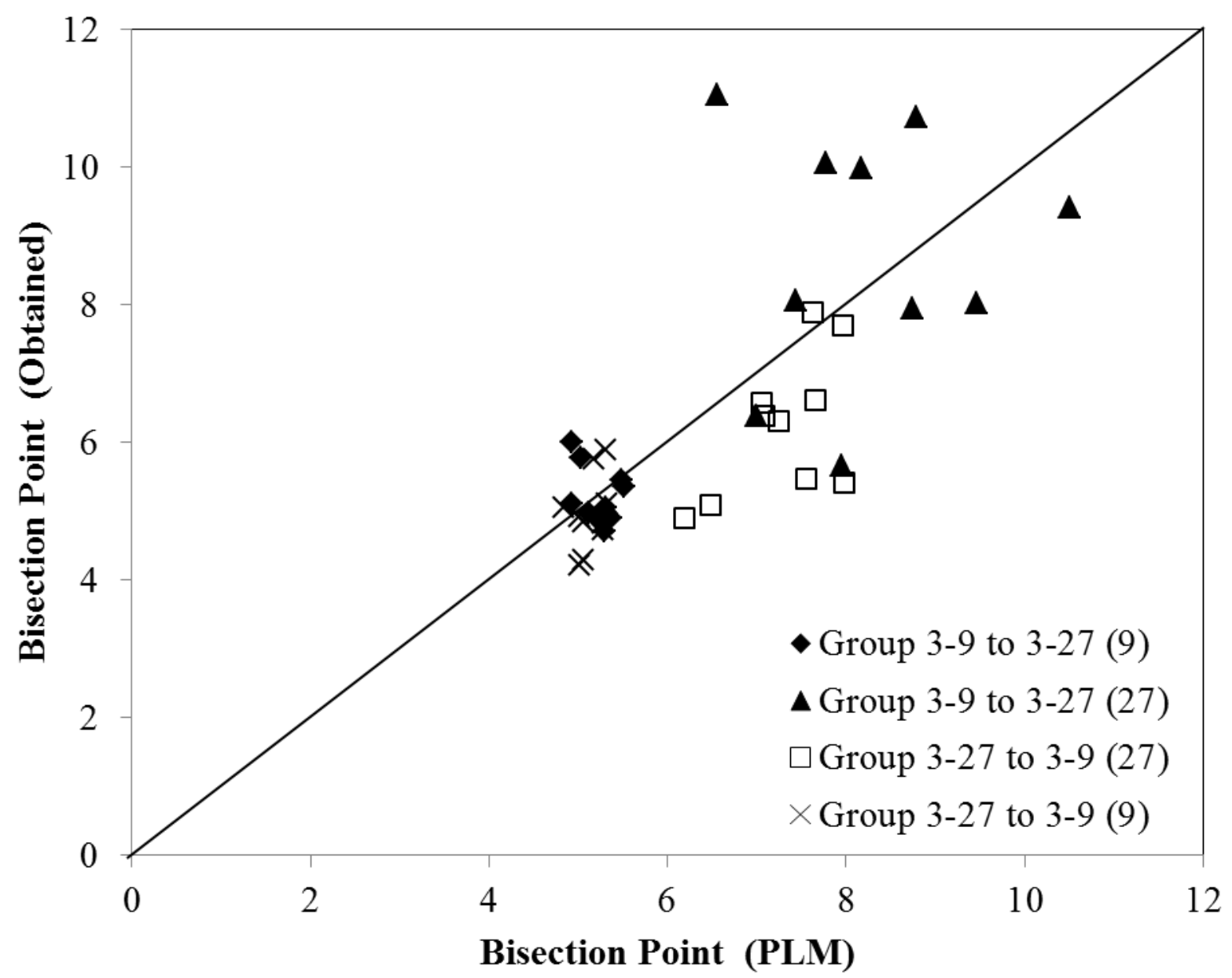

Figure 8. Bisection point (obtained) plotted against bisection point (PLM) for each phase of each group in Exp. 2. The diagonal represents the line for no difference between the two measures. Values in parentheses represent the durations (s) of the long anchor. 

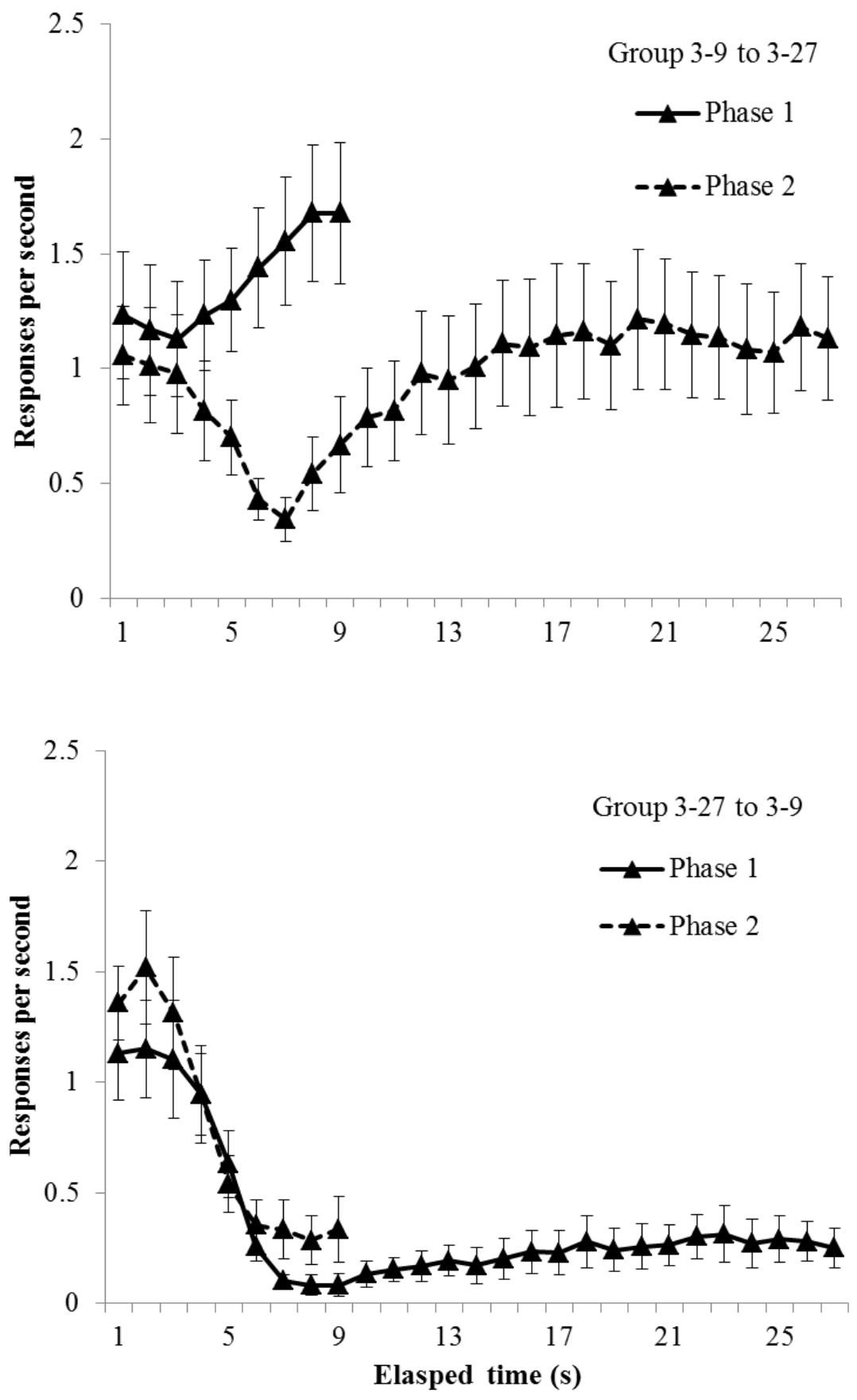

Figure 9. Group mean responses per second $( \pm S E M)$ on the long anchor sample cue as a function of elapsed time (s) for two phases in Exp. 2. The top graph refers to Group 3-9 to 3-27 and the bottom graph to Group 3-27 to 3-9. 

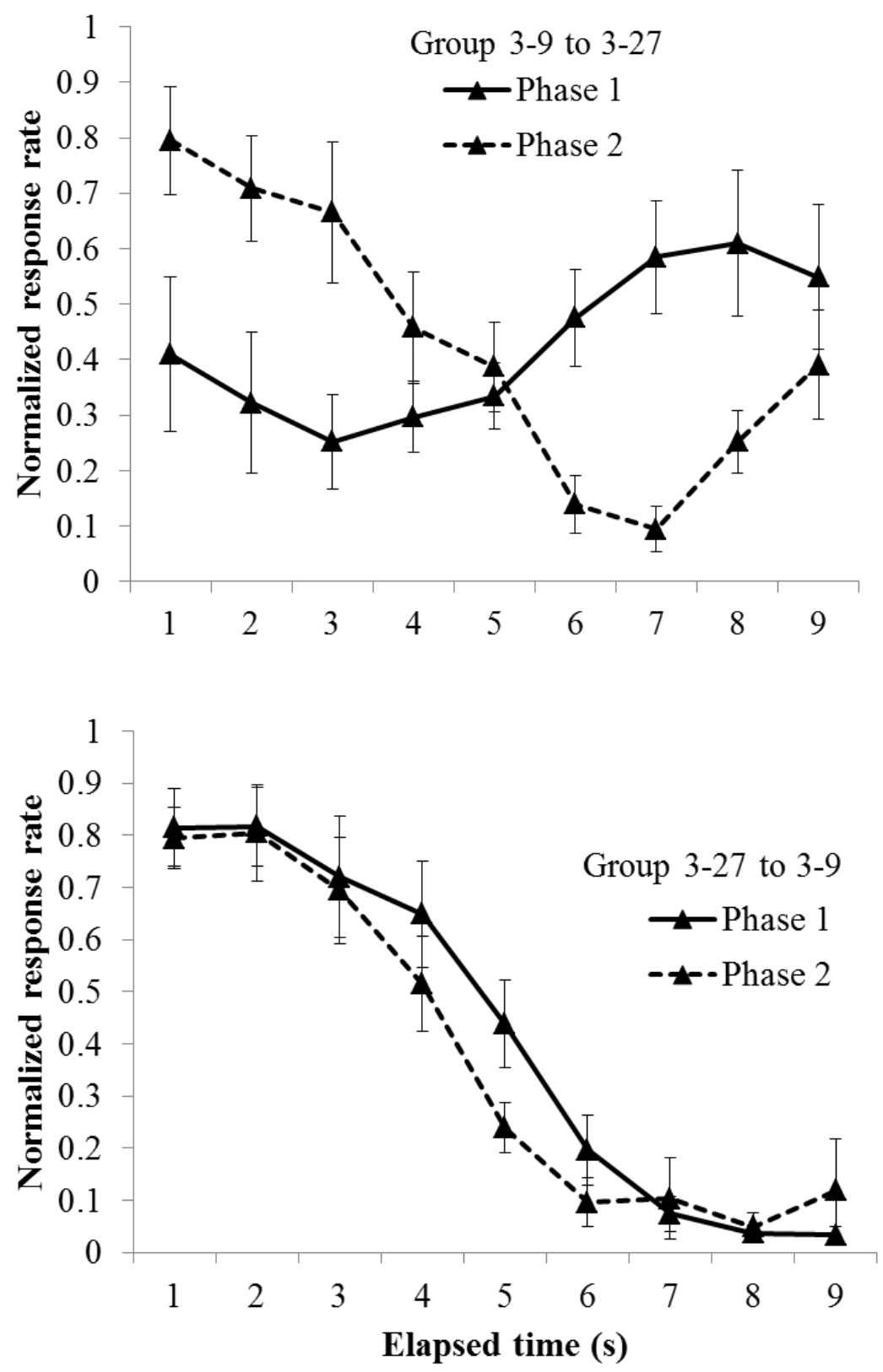

Figure 10. Group mean normalized response rate $( \pm S E M)$ at the sample key as a function of time during the first $9 \mathrm{~s}$ of the long anchor trials for each phase in Exp. 2. The top graph refers to Group 3-9 to 3-27 and the bottom graph to Group 3-27 to 3-9. 

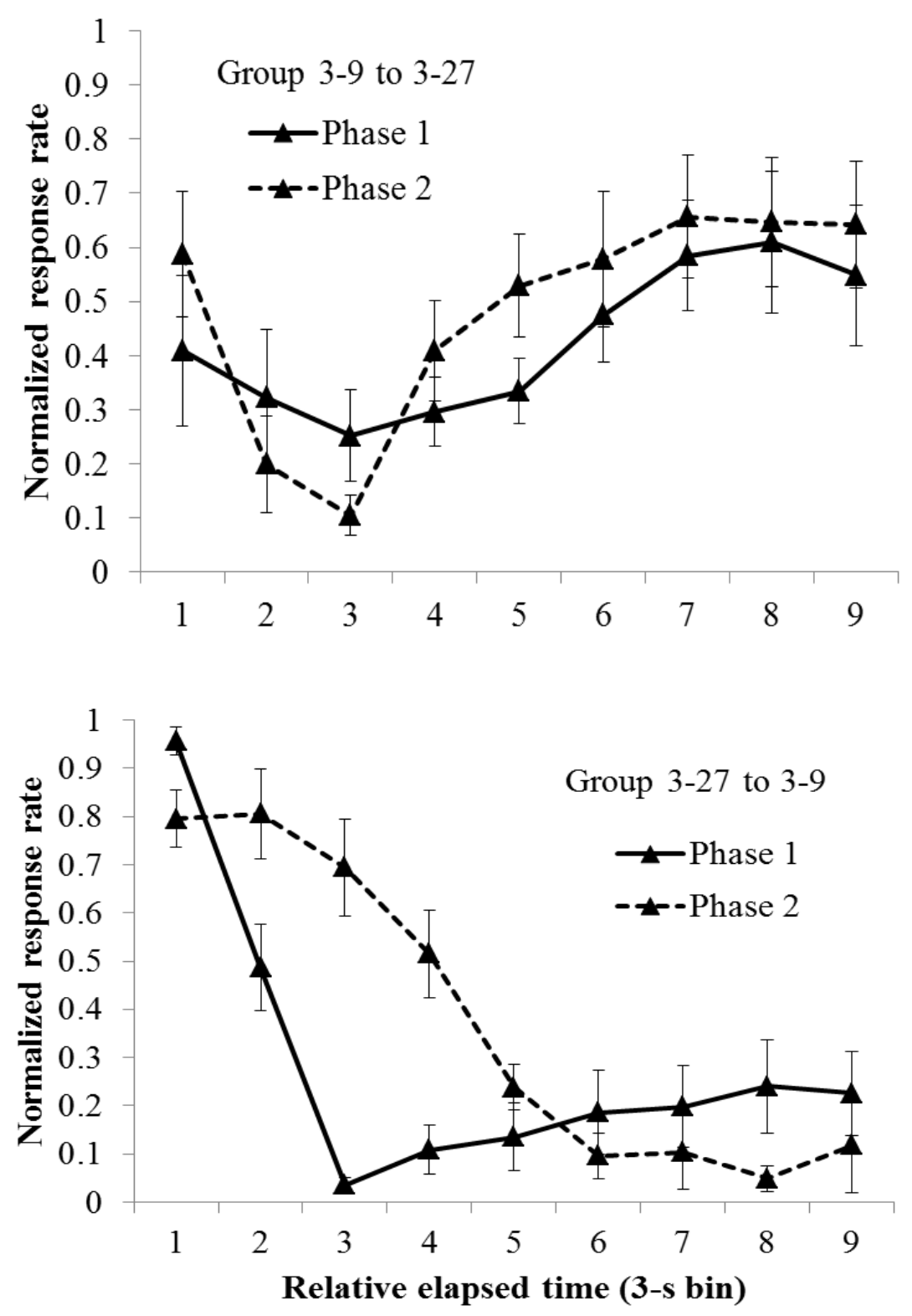

Figure 11. Group mean normalized response rate $( \pm S E M)$ as a function of relative elapsed time of the long anchor trials for both phases in Exp. 2. The top graph refers to Group 3-9 to 3-27 and the bottom graph to Group 3-27 to 3-9. 


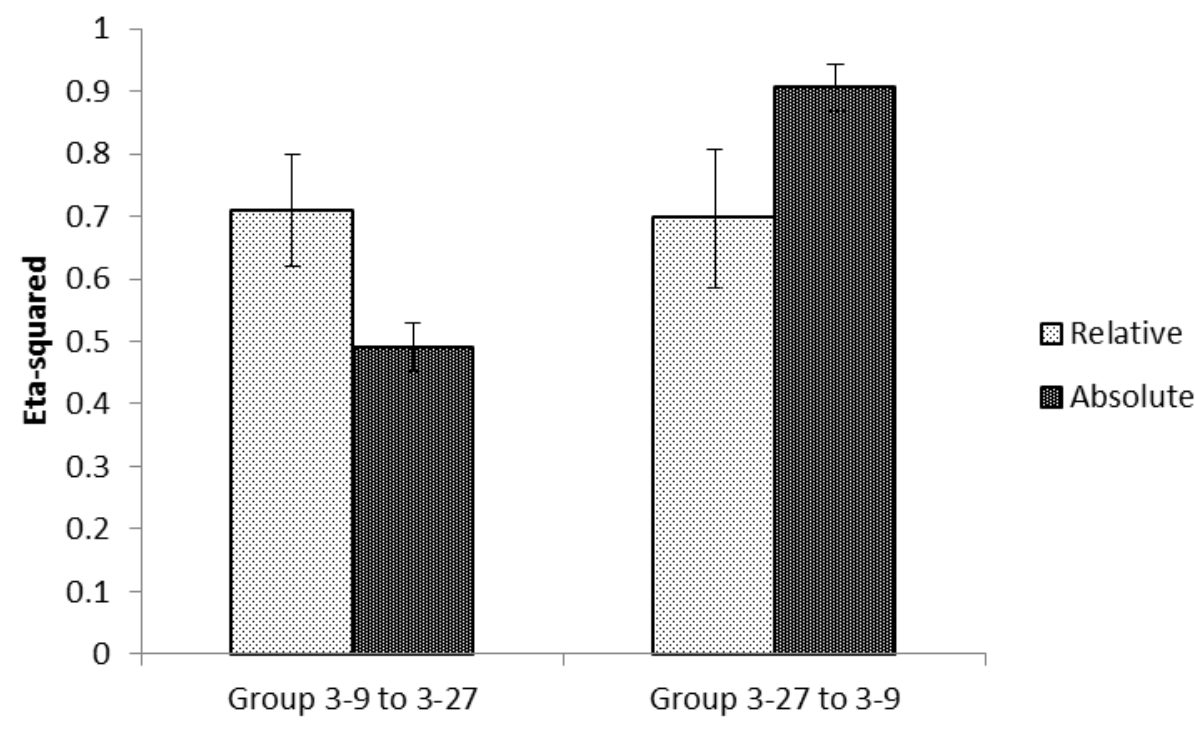

Figure 12. Group mean eta-squared $( \pm S E M)$ for the relative elapsed time scale and the absolute elapsed time scale for each group in Exp. 2. 

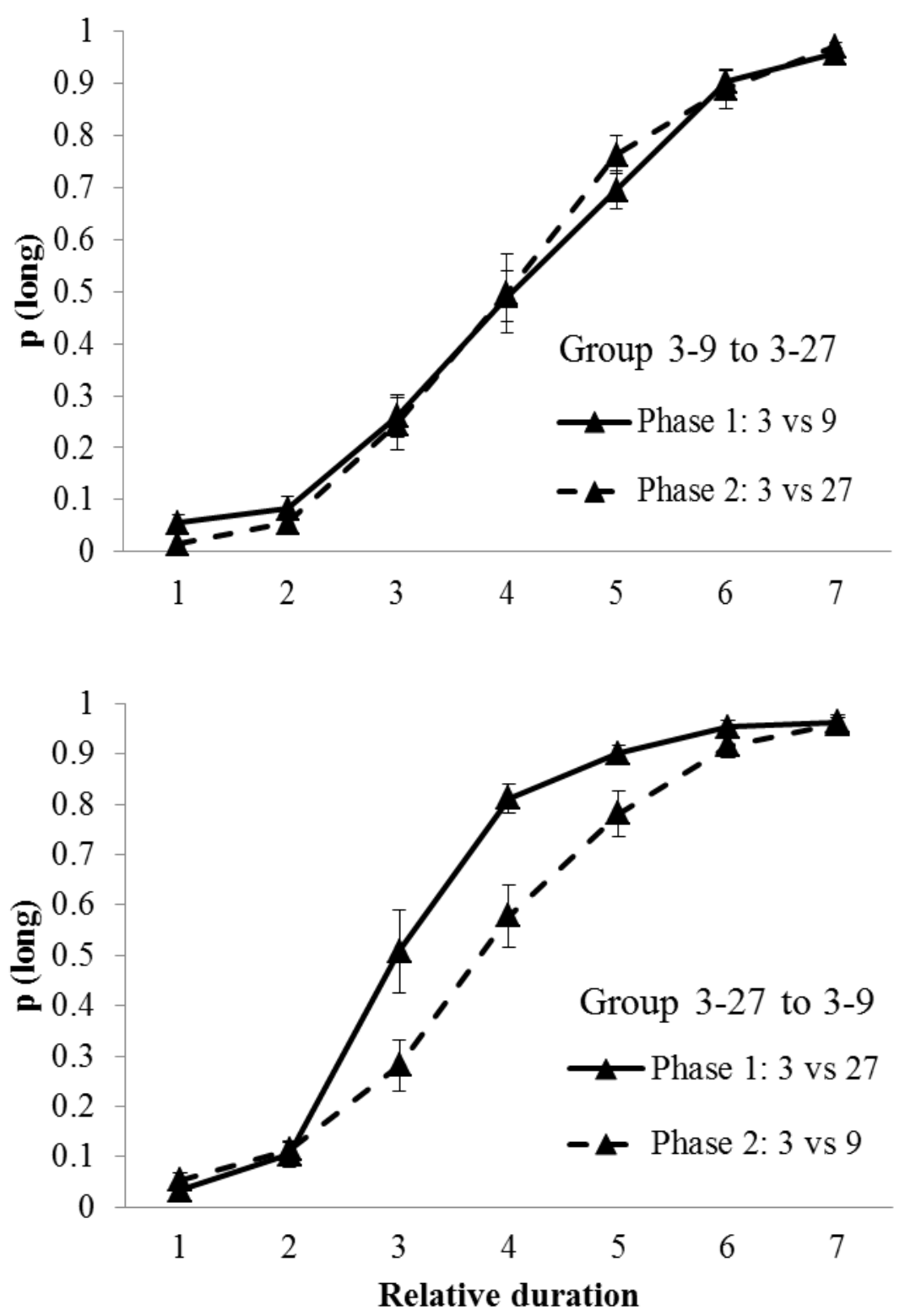

Figure 13. Group mean $\mathrm{p}$ (long) $( \pm S E M)$ as a function of the relative duration (log scale) for Phases 1 and 2 in Exp. 2. The upper and lower graphs refer to Group 3-9 to 3-27 and Group 3-27 to $3-9$, respectively. 


\section{Bibliography}

Allan, L. G. (2002). The location and interpretation of the bisection point. The Quarterly Journal of Experimental Psychology, Section B, 55(1), 43-60.

Brown, B. L., Hohn, S., Faure, A., von Horsten, S., Le Blanc, P., Desvignes, N., El Massioui, N., \& Doyère, V. (2011). Temporal sensitivity changes with extended training in a bisection task in a transgenic rat model. Frontiers in Integrative Neuroscience, 5. 44. doi:10.3389/fnint.2011.00044

Brown, B. L., Richer, P., \& Doyère, V. (2007). The effect of an intruded event on peak-interval timing in rats: Isolation of a postcue effect. Behavioural Processes, 74(3), 300-310. doi:10.1016/j.beproc.2006.11.004

Church, R. M. (1993). Human models of animal behavior. Psychological Science, 4(3), 170-173. doi: 10.1111/j.1467-9280.1993.tb00482.x

Church, R. M. (2002). A tribute to John Gibbon. Behavioural Processes, 57(2), 261-274. doi:10.1016/S0376-6357(02)00018-9

Church, R. M., \& Deluty, M. Z. (1977). Bisection of temporal intervals. Journal of Experimental Psychology: Animal Behavior Processes, 3(3), 216-228. doi:10.1037/00977403.3.3.216

Dews, P. B. (1962), The effect of multiple $S^{\Delta}$ periods on responding on a fixed-interval schedule. Journal of the Experimental Analysis of Behavior, 5(3), 369-374. doi: 10.1901/jeab.1962.5-369

Dews, P. B. (1970). The theory of fixed-interval responding. In W. N. Schoenfeld (Ed.), The theory of reinforcement schedules. New York: Appleton-Century-Crofts.

Dinsmoor, J. A. (1995). Stimulus control: Part II. The Behavior Analyst, 18(2), 253. 
Fantino, E. (1967). Preference for mixed- versus fixed-ratio schedules. Journal of the Experimental Analysis of Behavior, 10(1), 35-43. doi: 10.1901/jeab.1967.10-35

Ferster,C. B. \& Skinner, B. F. (1957). Schedules of reinforcement. Appleton-Century-Crofts, New York.

Gallistel, C. R. \& Gibbon, J. (2000). Time, rate, and conditioning. Psychological Review, 107(2), 289-344. doi: 10.1037/0033-295X.107.2.289

Gibbon, J. (1977). Scalar Expectancy Theory and Weber's Law in Animal Timing. Psychological Review, 84(3), 279-325. doi: 10.1037/0033-295X.84.3.279

Gibbon, J. (1981). On the form and location of the psychometric bisection function for time. Journal of Mathematical Psychology, 24(1), 58-87. doi:10.1016/0022-2496(81)900353

Gibbon, J., Baldock, M. D., Locurto, C., Gold, L., \& Terrace, H. S. (1977). Trial and intertrial durations in autoshaping. Journal of Experimental Psychology: Animal Behavior Process, 3(3), 264-284. doi: 10.1037/0097-7403.3.3.264

Grommet, E. K. (2013) The Role Of Clock And Memory Processes In The Timing Of Fear Cues In Humans. All Legacy Dissertations and Theses. Paper 121.

Honig, W. K. \& Urcuioli, P. J. (1981). The legacy of Guttman and Kalish (1956): 25 years of research on stimulus generalization. Journal of the Experimental Analysis of Behavior, 36(3), 405-445. doi: 10.1901/jeab.1981.36-405

Killeen, P. \& Fetterman, J. G. (1988). A Behavioral Theory of Timing. Psychological Review, 95(2), 274-295. doi: 10.1037/0033-295X.95.2.274 
Killeen, P., Fetterman, G., \& Bizo, L. A. (1997). Time’s causes. In: Bradshaw, C. M., \& Szabadi, E. (Eds.), Time and Behaviour: Psychological and Neurobiological Analyses. Elsevier Science Publishers, Amsterdam, pp. 79-131.

Machado, A. (1997). Learning the temporal dynamics of behavior. Psychological review, 104(2), 241-265. doi: 10.1037/0033-295X.104.2.241

Machado, A. \& Keen, R. (2003). Temporal discrimination in a long operant chamber. Behavioural Processes, 62(1), 157-182. doi:10.1016/S0376-6357(03)00023-8

Meck, W. H. (1983). Selective adjustment of the speed of the internal clock and memory processes. Journal of Experimental Psychology: Animal Behavior Processes, 9(2), 171-201. doi: 10.1037/0097-7403.9.2.171

Meck, W. H. \& Church, R. M. (1983). A mode control model of counting and timing processes. Journal of Experimental Psychology: Animal Behavior Processes, 9(3), 320-334. doi: $10.1037 / 0097-7403.9 .3 .320$

Platt, J. R., \& Davis, E. R. (1983). Bisection of temporal intervals by pigeons. Journal of Experimental Psychology: Animal Behavior Processes, 9(2), 160-170. doi: 10.1037/0097-7403.9.2.160

Raslear, T. G. (1983). A test of the Pfanzagl bisection model in rats. Journal of Experimental Psychology: Animal Behavior Processes, 9(1), 49-62. doi: 10.1037/0097-7403.9.1.49 Raslear, T. G. (1985). Perceptual bias and response bias in temporal bisection. Perception and Psychophysics, 38(3), 261-268. doi: 10.3758/BF03207153

Richelle, M., \& Lejeune, H. (1980). Time in animal behaviour. Oxford:Pergamon. Roberts, S. (1981). Isolation of an Internal Clock. Journal of Experimental Psychology: Animal Behavior Processes, 7(3), 242-268. doi: 10.1037/0097-7403.7.3.242 
Siegel, S. F. (1986). A test of the similarity rule model of temporal bisection. Learning and Motivation, 17(1), 59-75. doi:10.1016/0023-9690(86)90020-2

Staddon, J. E., \& Simmelhag, V. L. (1971). The" supersitition" experiment: A reexamination of its implications for the principles of adaptive behavior. Psychological Review, 78(1), 3-43. doi: $10.1037 / \mathrm{h} 0030305$

Stubbs, D. A. (1968). The discrimination of stimulus duration by pigeons. Journal of the Experimental Analysis of Behavior, 11(3), 223-238. doi:10.1901/jeab.1968.11-223

Stubbs, D. A. (1976). Scaling of stimulus duration by pigeons. Journal of the Experimental Analysis of Behavior, 26(1), 15-25. doi: 10.1901/jeab.1976.26-15

Wearden, J. H. (1991). Human performance on an analogue of an interval bisection task. The Quarterly Journal of Experimental Psychology Section B: Comparative and Physiological Psychology, 43B(1), 59-81.

Zentall, T. R., Friedrich, A. M., \& Clement, T. S. (2006). Required pecking alters judgments of the passage of time by pigeons. Psychonomic Bulletin \& Review, 13(6), 1038-1042. doi: 10.3758/BF03213922

Zentall, T. R. \& Singer, R. A. (2008). Required pecking and refraining from pecking alter judgments of time by pigeons. Learning \& Behavior, 36(1), 55-61. doi: 10.3758/LB.36.1.55 